\title{
Sound Scattering by Cylinders of Noncircular Cross Section Daniel DiPerna
}

B. S. Lafayette College (1987)

\section{Submitted in Partial Fulfillment of the Requirements for the Degree of \\ Doctor of Philosophy}

\author{
at the \\ at the
Massachusetts Institute of Technology and the

\section{Woods Hole Oceanographic Institution} \\ October 1993 \\ (c) Daniel T. DiPerna (1993) \\ The author hereby grants to MIT and WHOI permission to reproduce and \\ distribute paper or electronic copies of this thesis in whole or in part.
}

\begin{tabular}{|c|} 
MARINE \\
BIOLOGICAL \\
LABORATORY \\
\hline LIB RARY RY \\
\hline $\begin{array}{c}\text { WOZRS HOLE, MASS. } \\
\text { W. H. O. I. }\end{array}$ \\
\hline
\end{tabular}

Signature of Author

Department of Applied Ocean Physics and Engineering October 15, 1993

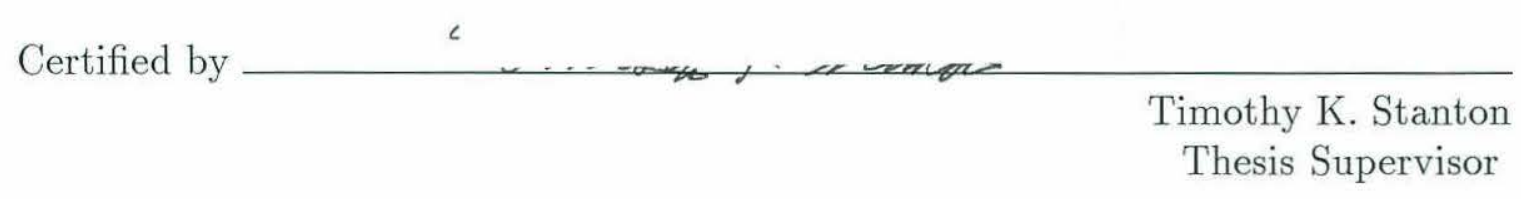

Accepted by

Arthur Baggeroer

Chairman, Joint Committee for Applied Ocean Science and Engineering, Massachusetts Institute of Technology - Woods Hole Oceanographic Institution

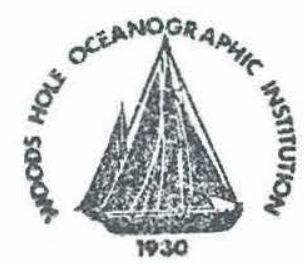




\section{Sound Scattering by Cylinders of Noncircular Cross Section}

\section{Daniel DiPerna \\ Abstract}

This thesis investigates a new method for predicting the farfield scattered pressure of a plane wave due to an infinite cylinder of noncircular cross section. Both impenetrable and penetrable fluid boundary conditions will be treated for several types of cross sections and for a large frequency range. This new method requires the conformal mapping of both the exterior and interior of a closed surface to a semi-infinite strip. Numerically efficient algorithms will be presented for both of these cases.

A new method for satisfying the boundary conditions will be developed, as well as an efficient algorithm for generating the required modal functions on the boundary. Numerical results are presented for cross sections in the shape of an ellipse, square, and three leaf clover. In all cases, the results compare extremely well with exact or high frequency asymptotic results. 


\section{Acknowledgements}

There are many people who are directly or indirectly responsible for the successful completion of this thesis. First I would like to thank my thesis advisor Tim Stanton, who allowed me to pursue my own ideas and encouraged me when things were not going well.

On my thesis committee are Prof. Rob Fricke of MIT, who acted as a sounding board for me for many years, and Prof. Yue-Ping Guo, whose contributions came at a later stage of my research, but were extremely important to the successful completion of this thesis.

I would also like to thank Dr. Dajun Tang of WHOI, who served as my thesis defense chairman, as well as being an inspiration to me by being so enthusiastic about science.

I thank Jake Peirson, Abbie Jackson and the rest of the WHOI Education Office staff for their help in the administrative portion of my graduate study.

I would also like to give special thanks to Bob Eastwood who not only helped me do battle with the computer, but also provided, at times, a place for me to live and a constant distraction which certainly added much to this thesis (time, not content.)

There are many others who contributed to my education in general over the past few years. Among these are my fellow students Franz Hover, Ram Gopalkrishnan, Kevin LePage, and Chick Corrado, as well as my friends from my soccer team Rui Correia, Abdul Faiky, and Carlos Santos, who were like family to me.

Most of all, I would like to thank my parents, without whose emotional support and encouragement I would never have completed this program. 


\section{Contents}

1 Introduction $\quad 10$

1.1 Review of Relevant Literature . . . . . . . . . . . . . . . . . . 13

1.1.1 Analytic Solutions . . . . . . . . . . . . . . 13

1.1.2 Approximate Solutions . . . . . . . . . . . . . 15

1.1.3 Numerical Solutions . . . . . . . . . . . . . . 16

1.2 Statement of the Problem . . . . . . . . . . . . . . 17

1.3 Outline of Thesis . . . . . . . . . . . . . . . . . 19

2 Analytic Development 20

2.1 Conformal Mapping . . . . . . . . . . . . . . . . 23

2.1 .1 Exterior mapping . . . . . . . . . . . . 26

2.1 .2 Interior Mapping . . . . . . . . . . . . . . . . . . . . 28

2.1.3 Example.......................... 29

2.2 Solutions of the Helmholtz Equation . . . . . . . . . . . . . . . 29

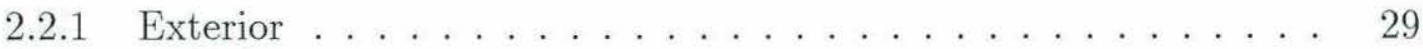


2.2 .2 Interior $\ldots \ldots \ldots \ldots \ldots \ldots \ldots \ldots \ldots \ldots \ldots$

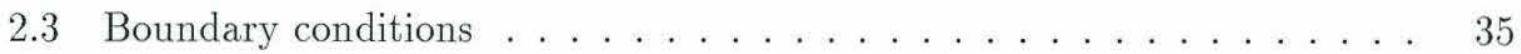

$\begin{array}{lll}3 & \text { Numerical Considerations } & 37\end{array}$

3.1 Boundary Conditions . . . . . . . . . . . . . . . . . . . . . 37

3.1.1 Example: Circular cylinder . . . . . . . . . . . . . . . . . 39

3.1 .2 Noncircular cylinder . . . . . . . . . . . . . . . . . . 41

3.2 Convergence . . . . . . . . . . . . . . . . . . . . . . . 43

3.3 Numerical Computation of Modal Functions . . . . . . . . . . . . . 45

4 Numerical Results $\quad 49$

4.1 Numerical Efficiency . . . . . . . . . . . . . . . . . . . . . . . . . 49

$4.2 \quad$ Ellipse . . . . . . . . . . . . . . . . . . . . . 51

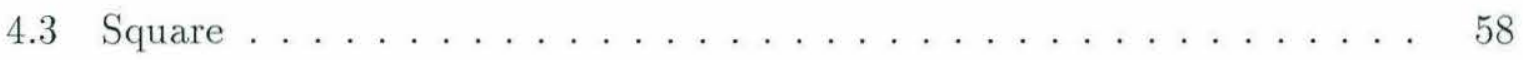

4.4 Three Leaf Clover . . . . . . . . . . . . . . . . . . . . . . . . 62

5 Discussion and Conclusions $\quad 70$

5.1 Conclusions . . . . . . . . . . . . . . . . . . . . 70

5.2 Recommendations for Future Research . . . . . . . . . . . . . . 72

$\begin{array}{lll}6 & \text { Appendices } & 73\end{array}$

6.1 Appendix A: Numerical Solution of Nonlinear System of Equations _ . . 73 
6.2 Appendix B: Equations used in Numerical comparisons . . . . . . . . 76

6.2.1 Exact Solutions for Separable Geometries . . . . . . . . . . 76

6.2.2 High Frequency (Physical Optics) Approximation . . . . . . . 81

6.2.3 Distorted Wave Born Approximation . . . . . . . . . . . 82 


\section{List of Figures}

2.1 Scattering Geometry . . . . . . . . . . . . . . . . . 21

2.2 Invalid scatterer geometry $\ldots \ldots \ldots \ldots \ldots \ldots \ldots \ldots$

2.3 Example of interior and exterior mapping: (a) curves $u=$ constant for several values of $u$, with the inner curve showing $u=0$ along with the original parameterization in circular coordinates. (b) curves $\zeta=$ constant for several values of $u$, with the outer curve showing $\zeta=0$ along with the original parameterization in circular coordinates. . . . . . . . 30

2.4 Contours of Integration . . . . . . . . . . . . . . . . . 33

4.1 Backscattered form function of a Rigid Ten to One Ellipse: $\theta_{0}=0$. The solid curve is from the FMM using 16 modes, the dashed curve uses cylindrical weighting functions using 16 modes, and the dash-dotted curve uses cylindrical weighting functions using 22 modes. . . . . . . . 52

4.2 Geometry of Cross section of Elliptic Cylinder . . . . . . . . . . . 54

4.3 Backscattered form function of a Rigid Ten to One Ellipse: $\theta_{0}=0$ (direction of incidence along the major axis). Both exact solution and approximate solution from the FMM are plotted. . . . . . . . . 55 
4.4 Backscattered form function of a Rigid Ten to One Ellipse: $\theta_{0}=\frac{\pi}{2}$ (direction of incidence along the minor axis). Both exact solution and approximate solution from the FMM are plotted. . . . . . . . . . . . . 56

4.5 Backscattered form function of a Rigid Two to One Ellipse: $\theta_{0}=0$ (direction of incidence along the major axis). Both exact solution and approximate solution from the FMM are plotted. . . . . . . . . . . .

4.6 Backscattered form function of a Penetrable Two to One Ellipse: $\theta_{0}=0$ (direction of incidence along the major axis). Both exact solution and approximate solution from the FMM are plotted. The material parameters are $\frac{\rho_{1}}{\rho}=2$ and $\frac{c_{1}}{c}=1.05 \ldots \ldots \ldots \ldots \ldots \ldots \ldots \ldots \ldots \ldots \ldots \ldots \ldots \ldots$

4.7 Geometry of Cross Section of a Square Cylinder . . . . . . . . . . .

4.8 Backscattered form function of a Rigid Square : $\theta_{0}=\frac{\pi}{4}$ (direction of incidence from the corner of the square). The dashed curve is from the physical optics approximation, and the solid curve is from the FMM under rigid boundary conditions. . . . . . . . . . . . . . .

4.9 Backscattered form function of a Soft Square : $\theta_{0}=\frac{\pi}{4}$ (direction of incidence from the corner of the square). The dashed curve is is from the physical optics approximation, and the solid curve is from the FMM under soft boundary conditions . . . . . . . . . . . . . . . .

4.10 Backscattered form function of a Rigid Square : $\theta_{0}=0$ (direction of incidence from the side of the square). The dashed curve is is from the physical optics approximation, and the solid curve is from the FMM under soft boundary conditions. . . . . . . . . . . . . . . 
4.11 Backscattered form function of a Weakly Scattering Square : $\theta_{0}=0$ (direction of incidence from the side of the square). The dashed curve is from the DWBA, and the solid is from the FMM. The material parameters are $\frac{\rho_{1}}{\rho}=1.05$ and $\frac{c_{1}}{c}=1.05 \ldots \ldots \ldots \ldots$

4.12 Geometry of Cross Section of Three Leaf Clover . . . . . . . . . . . . .

4.13 Backscattered form function of a Rigid Clover : $\theta_{0}=\pi / 6$. The dashed curve is from the physical optics approximation, and the solid curve is from the FMM under soft boundary conditions. . . . . . . . . . . . .

4.14 Backscattered form function from a 2:1 Ellipse. $\theta_{0}=0$. The solid curve is is from the physical optics approximation, the dashed curve is from the exact solution under soft boundary conditions, and the dotted curve is from the exact solution under rigid boundary conditions. This illustrates the fact that the physical optics solution will not necesarily accurately predict the level of the nulls. . . . . . . . . . . . . . . 


\section{Chapter 1}

\section{Introduction}

An important part of underwater acoustics research deals with how sound interacts with different boundaries. There are a wide variety of boundaries that exist in the ocean environment. There are two dimensional surfaces such as the ocean surface and ocean bottom, as well as three dimensional boundaries such as fish or submarines. Another classification of the boundary is the material composition of the surface. For example, a water sediment bottom scatters sound differently than the water air interface. How sound interacts with these different types of boundaries is important in the design of any sonar system. It is important to quantify how these different boundaries or objects scatter sound, whether it be for the detection of an object such as a submarine, or for an inverse problem, such as estimating biomass of marine organisms.

The manner and degree to which an object scatters sound can depend strongly on the shape of the scatterer, as well as on the relative size of the wavelength of the sound wave to the dimension of the scatterer. Exact analytical solutions can be found for only a

limited class of surfaces where separation of variables is applicable. This requires that the problem be formulated in a coordinate system in which the locus of points corresponding to the radial coordinate equal to a constant coincides exactly with the scatterer surface, and that the Helmholtz equation in that coordinate system is separable. For the problem 
treated in this thesis-that of an infinitely long cylinder of arbitrary cross section, these requirements are met only for three cross sections, circular, elliptic, and parabolic.

If separation of variables is not applicable for a particular shape, there are a wide variety of methods which may be used. All of these methods suffer from limitations on either the frequency range or the class of surfaces to which they are applicable. For example, one analytic approach that is formally exact is the perturbation method. This may be used for penetrable fluid or impenetrable boundary conditions, but is only valid if the shape is close to one of the separable geometries. One approximate solution is physical optics. However, it is valid only at high frequencies (i.e. wavelength much smaller than any dimension of the scatterer) and cannot acount for the penetrability of the scatterer.

In order to solve a problem over a wide range of frequencies and complex shapes, one must resort to numerical techniques. Although numerical methods which are formally exact have been developed, they can suffer from computational difficulties when implemented. For example, the Transition or T-Matrix method is a numerical technique which is used extensively. In practice when the surface deviates strongly from one of the separable geometries, the resulting matrix that needs to be inverted becomes illconditioned. Other numerical methods, such as the mode matching method (MMM) may be numerically intensive for even intermediate frequencies.

As can be seen from the above, there are many different approaches applied to the problem of sound scattering by cylinders of noncircular cross section. In general, problems arise when the scatterer surface differs significantly from a geometry in which the Helmholtz equation is separable. What is missing is a numerically efficient method that works over a wide frequency range for a large class of surfaces and for penetrable as well as impenetrable boundary conditions. The method presented in this thesis, the Fourier Matching Method (FMM) fills this void. An overview of the existing methods and the FMM is given in the following table. 


\begin{tabular}{||l||l|l|l|l|l||}
\hline & $\begin{array}{l}\text { Sep. of } \\
\text { Vars. }\end{array}$ & $\begin{array}{l}\text { Pert- } \\
\text { urbation }\end{array}$ & $\begin{array}{l}\text { T- } \\
\text { Matrix }\end{array}$ & MMM & FMM \\
\hline $\begin{array}{l}\text { Class of } \\
\text { Surfaces }\end{array}$ & $\begin{array}{l}\text { Limited } \\
\text { 3-Geom. }\end{array}$ & $\begin{array}{l}\text { Near } \\
\text { Separable }\end{array}$ & All & All & All \\
\hline $\begin{array}{l}\text { Comp. } \\
\text { Intensity }\end{array}$ & Low & Low & Mod. & High & Mod. \\
\hline $\begin{array}{l}\text { Freq. } \\
\text { Range }\end{array}$ & All & All & $\begin{array}{l}\text { Low- } \\
\text { Med. }\end{array}$ & $\begin{array}{l}\text { Low- } \\
\text { Med. }\end{array}$ & $\begin{array}{l}\text { Low- } \\
\text { Med. }\end{array}$ \\
\hline $\begin{array}{l}\text { Restric- } \\
\text { tions }\end{array}$ & None & $k \delta<<1$ & $\begin{array}{l}\text { Near } \\
\text { Separable }\end{array}$ & None & $\begin{array}{l}\text { two- } \\
\text { dimensional }\end{array}$ \\
\hline
\end{tabular}

The FMM consists of the following procedure:

1. Formulate the problem in a new coordinate system in which the radial coordinate $u=$ constant is the scatterer surface,

2. express the solution for the scattered field in terms of cylindrical eigenfunctions, and

3. solve for the unknown scattered field coefficients by expanding the boundary conditions in a Fourier series, and then forcing the first $M$ terms to be zero.

Step 1 is done because it has several desirable consequences. By using a special type of change of variables, a conformal mapping, calculation of the normal derivative of the pressure (which is required for calculating the normal particle velocity) becomes proportional to the derivative with respect to $u$ of the pressure. Another benefit is that equispacing sampling points in the new angular variable $v$ samples points on the surface which change rapidly at a higher spatial rate. This latter benefit has the effect of requiring fewer sampling points, therefore improving the numerical efficiency. The only 
drawback to the conformal mapping is that since conformal mapping is a two-dimensional procedure, the class of surfaces that the FMM can treat is limited to two-dimensional scatterers.

Step 2 assures that the Helmholtz equation is satisfied. This type of expansion may be used independently of scatterer shape, as opposed to the T-Matrix where the expansion functions depend on shape. The benefit of expressing the solution in this manner is that the cylindrical eigenfunctions (Hankel functions and complex exponentials) are readily available in standard math libraries, as opposed to Mathieu functions (eigenfunctions of the Helmholtz equation in elliptic coordinates), which are not readily available.

Step 3 results in a set of linear equations that are solved for the unknown coefficients. By satisfying the boundary conditions using this manner, the number of coefficients required to achieve a converged farfield solution is much smaller than the MMM.

\subsection{Review of Relevant Literature}

There has been extensive research done on this classic problem (for example see $[1,2,3])$. This section will give a brief overview of the existing methods that deal with the problem stated above, along with their regions of applicability and shortcomings. The three distinct types of methods are exact analytic solutions, approximate solutions, and numerical solutions.

\subsubsection{Analytic Solutions}

The scattering of sound by an infinitely long cylinder can only be solved exactly when the Helmholtz equation appropriate for that geometry is separable. This means that by assuming that the solution is a product of a function of the angular variable 
and a function of the radial variable, the Helmholtz equation for that specific geometry may be turned into two ordinary differential equations. Each of these ordinary differential equations depends on the same separation constant, and may be solved only if the constant is a characteristic value or eigenvalue. There are infinitely many of these eigenvalues, with each eigenvalue leading to a solution of the Helmholtz equation. One very desirable property is that the angular eigenfunctions form a complete, orthogonal set, so any function may be represented as a weighted sum of these functions. The total solution then is an infinite summation of these solutions, with an expansion coefficient associated with each solution.

Separation of variables is a very powerful solution technique, but in two dimensions the number of separable geometries is small, namely circular, elliptical, and parabolic[4]. For a circular cylinder, the problem may be solved exactly for impenetrable, penetrable fluid, and elastic boundary conditions $[5,6,7,8]$. The solution of the ordinary differential equations in this coordinate system is straightforward. The eigenvalues are simply integers, leading to the set of complex exponentials for the angular eigenfunctions and Bessel functions for the radial eigenfunctions. An important point here is that the eigenvalues are independent of wavenumber, which means that even for penetrable fluid boundary conditons, each scattered field coefficient is coupled only to one incident field coefficient.

For elliptical coordinates there is only a one-to-one coupling between scattered and incident field coefficients for impenetrable boundary conditions. Because the angular eigenfunctions depend on wavenumber, a closed form expression may be obtained only for impenetrable scatterers $[9,10]$. For penetrable scatterers, an expression involving the inverse of a matrix has been obtained by Yeh [11] by expanding the interior field in terms of the exterior eigenfunctions. The result of this is that each scattered field coefficient is coupled to all of the incident field coefficients. Porter [12] has obtained an approximate solution for weak scatterers with small eccentricity. An exact solution for elastic boundary conditions has not been found, although numerical results have been 
presented by Pillai et al [13] using the T-Matrix method. In Appendix B, separation of variables is discussed in detail for circular and elliptic coordinate systems, because this method provides a valuable check of the results generated by the method presented in this thesis.

Another type of analytic solution is a perturbation approach $[14,15]$. When the scatterer surface deviates only slightly from circular, the boundary conditions may be expanded in terms of a Taylor series. By equating orders of the perturbation parameter $\delta$, correction terms to the zeroth order solution (the circular solution) are obtained to any order of $\delta$. As in the case of the penetrable ellipse, each scattered field coefficient is coupled to all of the incident field coefficients, even for impenetrable scatterers. These correction terms are known analytically. The drawback of this method is that the perturbation series will only converge if $k \delta \ll 1$, where $k$ is the wavenumber of the incident wave.

\subsubsection{Approximate Solutions}

One class of approximate solutions is only valid at high frequency [16]. One such approximation is physical optics[17, 18, 19]. This approach starts with a Helmholtz integral formulation, and assumes that each point on the surface scatters as though it were part of a plane tangent to that point. The resulting integrals may be evaluated asymptotically by stationary phase or the saddle point method. This is a very powerful method because it yields insight into the physics involved in the scattering process, i.e. it shows which portions of the object contribute strongly to the scattered field. The limitations are that it cannot account for the penetrability of the scatterer or for diffraction from a sharp edge.

Another high frequency approximation which may take into account sharp edges is the geometric theory of diffraction $[20,21,22,23]$. The total scattered field is constructed 
by summing contributions from speculary reflected rays, surface waves, and diffracted rays. These contributions are obtained by analyzing canonical cases. For example, an edge diffraction contribution is calculated by analyzing the canonical case of diffraction from a wedge.

\subsubsection{Numerical Solutions}

There are various numerical solutions for the scattering of sound by a noncircular cylinder. One is a numerical solution of the integral equations $[24,25,26]$. An integral equation is written for the scattered field on the surface. This equation is then solved by discretizing the scattered field to yield a system of linear equations that may be solved for the unknown surface field. The major drawback of this method is that for high frequencies, the number of discretizations and thus unknowns becomes very large, making the method computationally intensive. There also may be spurious resonances corresponding to the interior eigenvalue problem.

Another numerical technique is the extended boundary condition or T-Matrix method $[27,3,28,13,29]$. This method assumes an expansion of the surface fields in terms of a complete set of orthogonal functions, and then uses the Helmholtz integral for-

mula to assure that the Helmholtz equation is satisfied. The result is that the boundary conditions will be met exactly, but in truncating a matrix, the Helmholtz equation is not satisfied perfectly. This can lead to problems for scatterers whose shapes deviate strongly from circular, as the matrices that need to be inverted tend to become ill-conditioned. There are various ways to deal with these shortcomings. For example Bates and Wall [28] suggested expanding the surface fields in terms of a more appropriate set of functions. For an elongated scatterer, it is desirable to expand the surface fields in terms of elliptic cylinder functions (Mathieu functions.) Varadan and Varadan [29] have also suggested an iterative method to alleviate the ill-conditioning for elongated scatterers. 
Another numerical method is the mode matching method (MMM) [30, 31]. The surface fields are expanded in terms of a series of solutions (modal functions) of the Helmholtz equation, with an unknown coefficient associated with each modal function. In contrast to the separation of variables technique, these individual solutions are not orthogonal. This makes the choice of the unknown scattered field coefficients far more difficult. The procedure used in the MMM is to choose the unknown coefficients to minimize the mean squared error in the boundary condition. This minimization is achieved by differentiating the mean squared error on the surface with respect to each scattered field coefficient, leaving a linear system of equations to be solved. This method can be proven to converge to the correct solution. However, a large number of terms are needed in general to match the boundary condition, when only a few terms are necesary to calculate the farfield scattered pressure. In general, most of the computation time is spent in calculating the modal functions, so there is an inefficiency in carrying more terms than are necesary to calculate the farfield scattered pressure.

\subsection{Statement of the Problem}

As discussed previously, much research has been conducted on the problem of sound scattering by an infinitely long cylinder of noncircular cross section. Each of these is valid for a very specific frequency range, boundary condition, or class of surfaces. In this thesis a new formulation will be presented that will efficiently generate farfield scattered pressure results for penetrable fluid or impenetrable infinitely long cylinders. The cross sections of the cylinders will involve ellipses, a square, and a three-leaf clover. These shapes fall into three major classes: elongated scatterers, scatterers with sharp edges, and scatterers with concavities, respectively. The formulation is the same regardless of surface type, for all of the surfaces mentioned above, with the surface fields being expanded in terms 
of circular eigenfunctions, as opposed to the T-Matrix method where the choice of basis functions depends on the shape.

The fact that the choice of basis function affects the numerical properties of the T-Matrix method is what motivated the research described herein. Bates and Wall suggested that the ill-conditioning problems for the T-Matrix could be alleviated by choosing a coordinate system in which the radial coordinate $=$ constant is as close to the scatterer as possible, and expanding the surface fields and Green's function in terms of the eigenfunctions in that new coordinate system. However, this still assumes that the scatterer surface will be close to a coordinate system that is separable, and requires the calculation of special functions such as Mathieu functions. One result of this research is that by expanding the surface fields in a Fourier series in the new angular variable $v$ and the Green's function in circular eigenfunctions, the T-Matrix will be able to handle even elongated scatterers without the ill-conditioning problems usually associated with this method.

In addition to using the circular eigenfunctions instead of more complicated special functions, the FMM has several desirable numerical properties. It facilitates and makes more efficient the calculation of the circular eigenfunctions and their derivatives on the scatterer surface. It also makes any numerical integration over the scatterer surface more efficient by sampling portions of the surface that are changing rapidly at a higher spatial rate than portions of the surface that are smooth. These issues are discussed in Chapter 2.

The major contributions of this thesis are:

1. An efficient algorithm for conformally mapping the exterior and interior of an arbitrary shape to a strip is developed and 
2. a new method for satisfying the boundary conditions is developed which improves the numerical efficiency of calculating the backscatter from noncircular cylinders and is valid for a large class of cross sections and frequencies.

\subsection{Outline of Thesis}

In Chapter 2 the class of surfaces that may be treated by the method presented in this thesis is discussed. Algorithms are then developed to conformally map the exterior and interior of a closed surface to a semi-infinite strip. It will be shown how the Helmholtz equation changes, and how to solve this new Helmholtz equation.

In Chapter 3, various numerical issues that arise in the solution of the scattering problem are discussed. These issues include how to satisfy the boundary condtions, how to truncate the resulting infinite series, and a numerically efficient way of calculating the modal functions and their normal derivatives on the surface.

In Chapter 4 the scattering results generated for a wide variety of surfaces and boundary conditions are presented. Included are results for high-aspect-ratio elliptic cylinders, a square cylinder, and a cylinder with concavities. Results are compared to exact solutions where applicable, or to approximate solutions.

In Chapter 5 there is a discussion of the method, and future research that could follow this thesis is addressed. 


\section{Chapter 2}

\section{Analytic Development}

The problem treated in this thesis is that of the scattering of a plane wave of sound by a penetrable fluid cylinder of non-circular cross section. The acoustic parameters (density and sound speed) of the obstacle are $\rho_{1}, c_{1}$, and those of the surrounding fluid are $\rho, c$. The geometry is shown in Fig.(2.1).

The approach used here is to take the problem formulated in Cartesian coordinates, and change variables (conformally map) to a new coordinate system in which the radial coordinate $=$ constant is the scatterer surface. This has the effect of making the boundary conditions, continuity of pressure and normal particle velocity, easier to satisfy, but it also makes the governing equation more complicated. However, due to the special form of the change of variables, the solution of the new equation is also obtainable.

Consider the wave equation

$$
\nabla^{2} P=\frac{1}{c^{2}} \frac{\partial^{2} P}{\partial t^{2}}
$$

where $P$ is pressure, $\nabla^{2}$ is the Laplacian operator, $c$ is the speed of sound, and $t$ is time. Using the form of the Laplacian operator in Cartesian coordinates and assuming a harmonic time dependence $e^{-i \omega t}$, where $\omega$ is the frequency (and suppressing it throughout this thesis), the wave equation becomes the Helmholtz equation:

$$
P_{x x}+P_{y y}+k^{2} P=0
$$




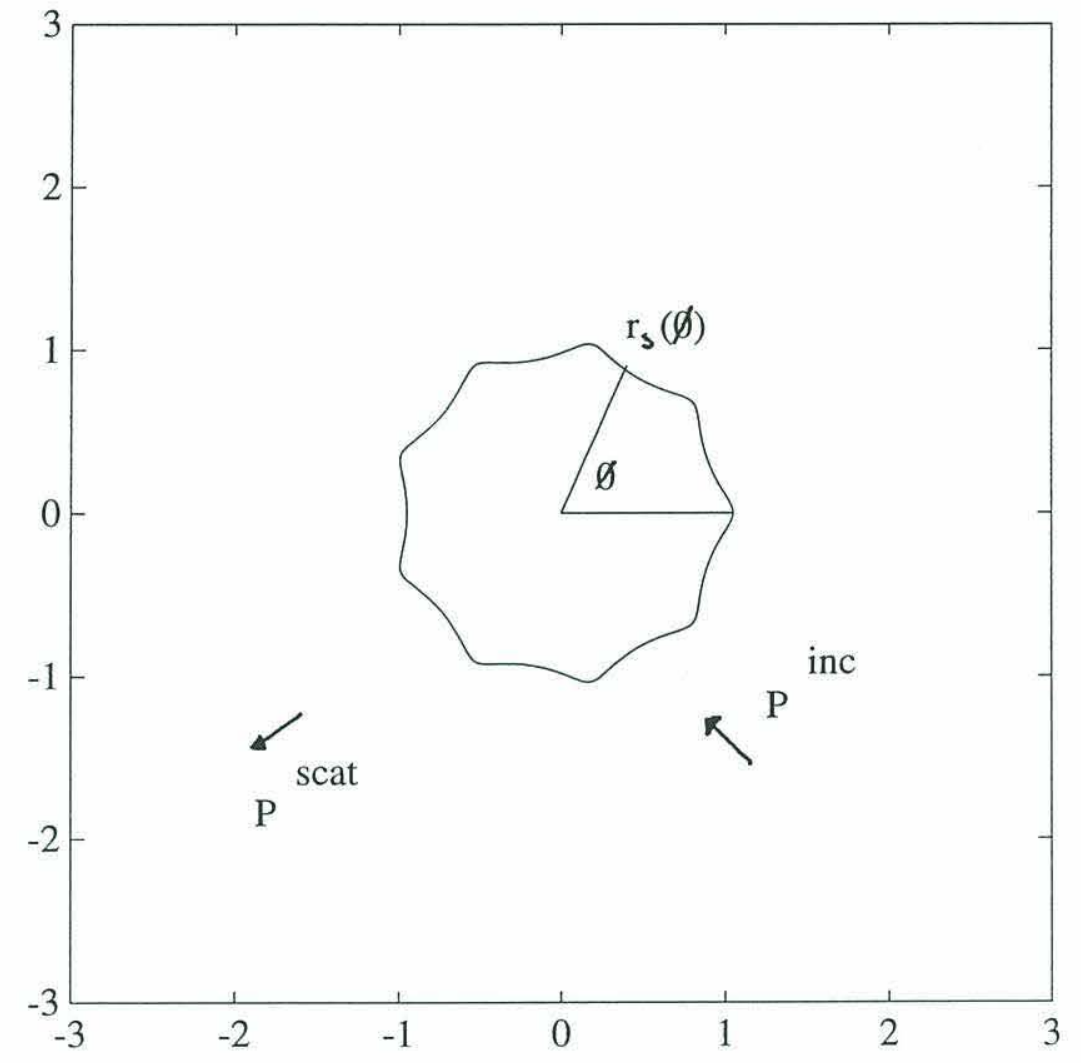

Figure 2.1: Scattering Geometry 
where $k=\omega / c$ is the wavenumber. Plane waves in the form $e^{i k(x \cos (\mu)+y \sin (\mu))}$ are solutions of this equation, where $\mu$ is the angle of propagation of the plane wave with respect to the $\mathrm{x}$-axis. Each plane wave is associated with an an amplitude $A(\mu), B(\mu)$. By integrating over the appropriate contours in $\mu$, expressions for the incident and scattered field may be found.

Changing to a new coordinate system changes the Helmholtz equation, giving the following:

$$
P_{u u}+P_{v v}+k^{2} \mathcal{F}(u, v) P=0
$$

where $(u, v)$ are the new coordinates, and $\mathcal{F}(u, v)$ is a function which depends on the shape of the scatterer. Note that this has the same form as the Helmholtz equation in Cartesian coordinates except that now the wavenumber is a function of position. Therefore, an analogous problem is wave propagation in a range and depth dependent media. This is a very difficult problem in general and will not be pursued in this thesis. However, by solving the scattering problem, a new technique for solving the propagation problem might be developed.

Solutions of this new Helmholtz equation can be found in terms of plane waves, but now the wavenumber is a function of position. By choosing the same contours of integration as in the circular case, solutions may be found in terms of cylindrical eigenfunctions. These integral representations, coupled with the fact that $u$ and $v$ are orthogonal, suggest a simple and efficient way to calculate the pressure and normal particle velocity, which are needed to satisfy the boundary conditions.

Changing to a new coordinate system also generates a new set of functions $e^{i n v}$. These are different than the exponentials that would ordinarily be generated by using cylindrical coordinates $\left(e^{i n \theta}\right)$. The new angular coordinate $v$ is a more natural way to describe the surface. By using this new set of functions, the boundary conditions are 
satisfied to some order of $e^{i n v}$, as well as satisfying the Helmholtz equation to the same order.

The remainder of this chapter is divided into two sections. The first shows how to conformally change variables. The second describes the resulting Helmholtz equation and its solutions.

\subsection{Conformal Mapping}

In this section it is shown how to conformally change variables to a new coordinate system in which the radial coordinate $=$ constant is the scatterer surface. This has several consequences. One is that the conformal nature of this change of variables ensures that the new coordinates are orthogonal. In other words, by changing from an $(x, y)$ coordinate system to a $(u, v)$ coordinate system, since $x$ and $y$ are orthogonal, $u$ and $v$ will also be orthogonal. This is a desirable property because it facilitates the computation of the pressure and the normal particle velocity on the boundary, which are needed to satisfy the boundary conditions. Also, by expressing the field quantities in terms of these more natural coordinates instead of circular cylindrical coordinates, points along the surface which change rapidly are sampled at a high spatial rate. For example, for a square cylinder, by choosing points equally spaced in angular coordinate $v$, points are sampled more rapidly near the corners where the field is expected to be changing more rapidly.

The following gives a procedure for generating the mapping function for both the exterior and the interior of the scatterer. Once these functions have been determined, the uniqueness of the map is checked for by determining where the Jacobian of the transformation is zero.

Consider the class of surfaces which may be described as follows:

$$
x=\rho_{s}(\phi) \cos (\phi)
$$




$$
y=\rho_{s}(\phi) \sin (\phi)
$$

where $\rho_{s}, \phi$ are circular cylindrical coordinates. The only constraint is that $\rho_{s}(\phi)$ must be single valued, i.e. for each $\phi$, there can be only one value of $\rho_{s}$ (see Fig.(2.2) for an example of a geometry that cannot be handled by this conformal mapping algorithm).

Without loss of generality, $\rho_{s}(\phi)$ may be expanded in a Fourier series in $\phi$ as follows:

$$
\rho_{s}(\phi)=a+\sum_{n=1}^{\infty}\left[r_{n}^{c} \cos (n \phi)+r_{n}^{s} \sin (n \phi)\right]
$$

where $a$ is the average radius, and $r_{n}^{c}$ and $r_{n}^{s}$ represent the deviation of the surface from circular. Collecting terms this may be rewritten as

$$
x+\left.i y\right|_{\text {surface }}=a e^{i \phi}+\sum_{n=1}^{\infty}\left[R_{n}^{*} e^{i(1+n) \phi}+R_{n} e^{i(1-n) \phi}\right]
$$

where

$$
R_{n}=\frac{1}{2}\left[r_{n}^{c}+i r_{n}^{s}\right]
$$

For a conformal map the following requirement must be met:

$$
x+i y=M(z) \quad z=u+i v
$$

where $M(z)$ is an analytic function, $u$ is the radial variable, and $v$ is the angular variable. The problem therefore is to choose an appropriate $M(z)$. A different $M(z)$ is needed for interior and exterior mappings. These mappings are different because one of the constraints on the mapping function is that the coordinate system becomes circular as the radial coordinate increases, and that the circles must be outside the surface. For the interior problem, a constraint is that as the radial coordinate decreases, the coordinate system becomes circular, and that the circles are inside the surface. Because of this difference, there are different forms for the mapping functions for the interior and exterior problems. 


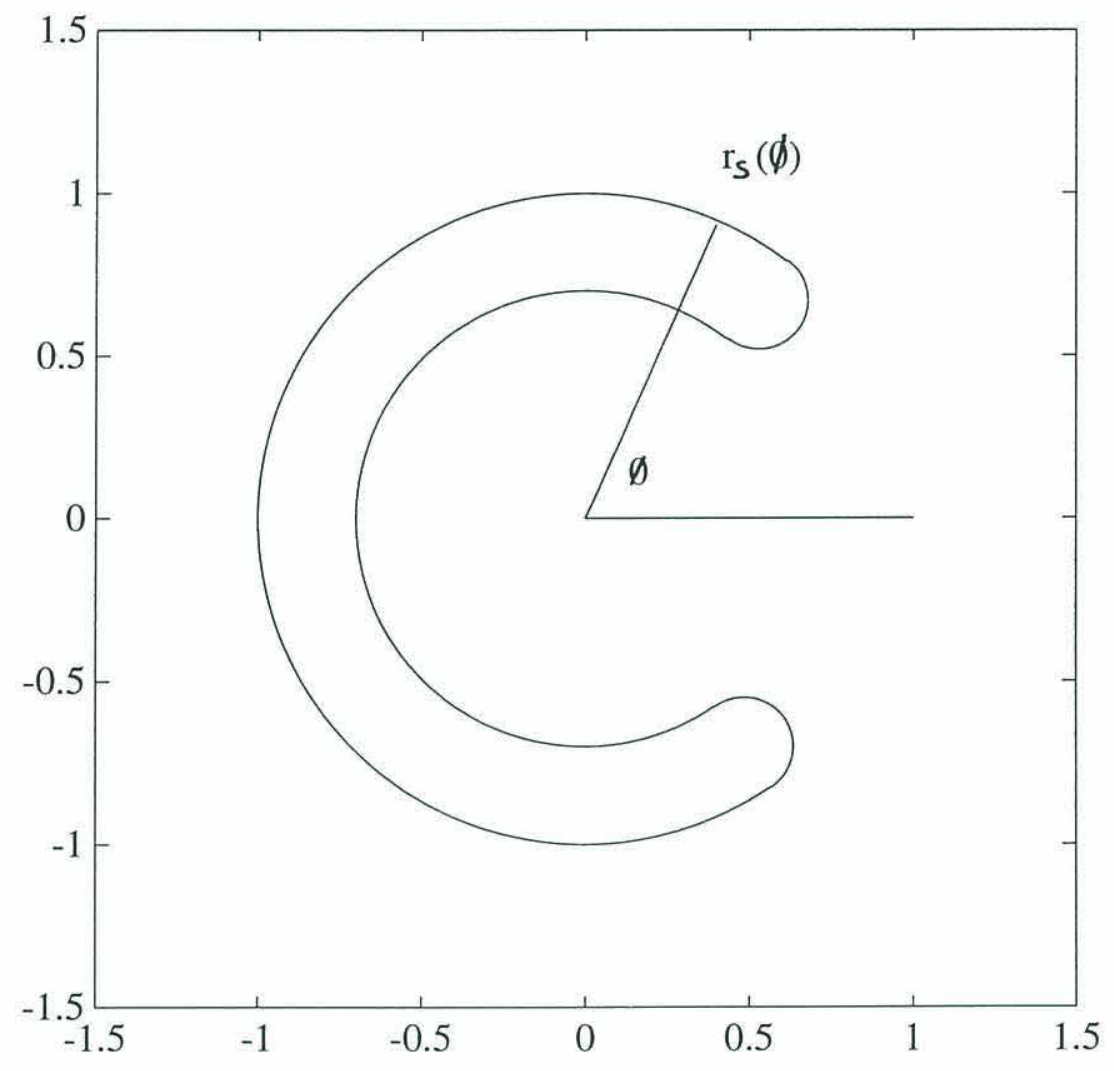

Figure 2.2: Invalid scatterer geometry 


\subsubsection{Exterior mapping}

Since a different mapping is needed for the interior and exterior problems, let the mapping function $M(z)$ be denoted by $G(z)$ for the exterior problem. The function $G(z)$ must be chosen so that the following three conditions are met:

1. As $u \rightarrow \infty$, the coordinate system is circular (this is necesary so that farfield results are directly comparable to existing results without having to perform a possibly very difficult inverse mapping),

2. the transformed Helmholtz equation is solvable, and

3. $u=0$ is the scatterer surface.

Assume the following form for the mapping function:

$$
G(z)=g_{-1} e^{z}+\sum_{n=0}^{\infty} g_{n} e^{-n z} .
$$

This form was chosen so that the mapping will automatically satisfy Conditions 1 and 2 .

Condition 1 is satisfied because as $u \rightarrow \infty, e^{-n z} \rightarrow 0$, leaving $G(z) \rightarrow g_{-1} e^{z}+g_{0}$, which is a circular coordinate system with center at $x=\operatorname{Real}\left(g_{0}\right), y=\operatorname{Imag}\left(g_{0}\right)$. Condition 2 is closely tied to the fact that the mapping must be unique, which will be discussed subsequently. Condition 3 may be satisfied by choosing the $g_{n}$ appropriately. To do this, the original parameterization in cylindrical coordinates of the surface is forced to be equal to the mapping function when the radial coordinate is zero, or:

$$
x+\left.i y\right|_{\text {surface }}=\left.G(z)\right|_{u=0}=g_{-1} e^{i v}+\sum_{n=0}^{\infty} g_{n} e^{-i n v}
$$

Comparing this with Eq.(2.6), it is seen that there is a fundamental difference. In Eq.(2.10) there are only negative frequency components $e^{-i n v}$ (other than $e^{i v}$ ), whereas in Eq.(2.6) there are both positive and negative frequency components, i.e. $e^{i(1+n) \phi}, e^{i(1-n) \phi}$. 
From this it is concluded that $\phi \neq v$. Now assume that

$$
\phi(v)=v+\sum_{l=1}^{\infty}\left[\delta_{l}^{s} \sin (l v)+\delta_{l}^{c} \cos (l v)\right] .
$$

This form was chosen so that if the surface were circular, the values of $\delta$ would all be zero, and $\phi=v$. Since the problem is periodic, we expect that the deviation of $\phi$ from $v$ should also be periodic, and therefore be representable by a Fourier series, which is what is inside of the summation.

The problem is now to choose the values of $\delta$ so that Eq.(2.6) and Eq.(2.10) are the same. Inserting Eq.(2.11) into Eq.(2.10) gives

$$
a e^{i \phi(v)}+\sum_{n=1}^{\infty}\left[R_{n}^{*} e^{i(1+n) \phi(v)}+R_{n} e^{i(1-n) \phi(v)}\right]=g_{-1} e^{i v}+\sum_{n=0}^{\infty} g_{n} e^{-i n v} .
$$

Multiplying both sides by $\frac{1}{2 \pi} e^{-i j v}$ and integrating over the range of $v$ leaves

$$
\frac{1}{2 \pi} \int_{0}^{2 \pi} e^{-i j v}\left\{a e^{i \phi(v)}+\sum_{n=1}^{\infty}\left[R_{n}^{*} e^{i(1+n) \phi(v)}+R_{n} e^{i(1-n) \phi(v)}\right]\right\} d v=\left\{\begin{array}{ll}
0 & j>1 \\
g_{j} & j \leq 1
\end{array} .\right.
$$

This comes from the orthogonality relationships of the complex exponential functions, i.e. $1 / 2 \pi \int_{0}^{2 \pi} e^{i n v} e^{-i m v} d v=\delta_{m, n}$, where $\delta_{m, n}$ is the Kronecker delta function. The upper result is a set of non-linear constraints that may be solved for the values of $\delta$. In practice this is done using an extension of the Newton-Raphson method (Appendix A). Once the values of $\delta$ are known, $\phi(v)$ is also known, and the lower result is used to calculate $g_{j}$.

Once the mapping coefficients are known, the uniqueness of the map must be checked for (i.e. that each $(u, v)$ corresponds to only one $(x, y))$. This is done by making sure that the Jacobian of the transformation is non-zero in the desired region (outside the scatterer.) This condition is

$$
\left|G^{\prime}(z)\right|^{2} \neq 0, \quad u \geq 0 .
$$




\subsubsection{Interior Mapping}

For the interior mapping, let $M(z)$ be denoted by $F(z)$, and $z=\zeta+i \eta$, where $\zeta$ is the radial coordinate, and $\eta$ is the angular coordinate. For the interior mapping, $F(z)$ must be chosen to satisfy the following conditions:

1. As $\zeta \rightarrow-\infty$, the coordinate system is circular,

2. the transformed Helmholtz equation is solvable, and

3. $\zeta=0$ is the scatterer surface.

Now assume a form for $F(z)$ as follows:

$$
F(z)=\sum_{n=0}^{\infty} f_{n} e^{n z}
$$

Again, Conditions 1 and 2 are automatically satisfied by the form of $F(z)$, and the $f_{n}$ must be chosen to satisfy the Condition 3 . Following the same procedure as for the exterior problem, let

$$
\phi(\eta)=\eta+\sum_{l=1}^{\infty}\left[\epsilon_{l}^{s} \sin (l \eta)+\epsilon_{l}^{c} \cos (l \eta)\right]
$$

where the values of $\epsilon$ must be determined. To do this let

$$
a e^{i \phi(\eta)}+\sum_{n=1}^{\infty}\left[R_{n}^{*} e^{i(1+n) \phi(\eta)}+R_{n} e^{i(1-n) \phi(\eta)}\right]=\sum_{n=0}^{\infty} f_{n} e^{i n \eta} .
$$

Multiplying both sides by $\frac{1}{2 \pi} e^{i j \eta}$ and integrating over the range of $\eta$ leaves

$$
\frac{1}{2 \pi} \int_{0}^{2 \pi} e^{i j \eta}\left\{a e^{i \phi(\eta)}+\sum_{n=1}^{\infty}\left[R_{n}^{*} e^{i(1+n) \phi(\eta)}+R_{n} e^{i(1-n) \phi(\eta)}\right]\right\} d \eta=\left\{\begin{array}{cc}
0 & j \geq 1 \\
f_{j} & j<1
\end{array} .\right.
$$

Again, the values of $\epsilon$ are solved for, and then values of $f_{j}$ are calculated .

For this map to be unique, the Jacobian must be non-zero inside the scatterer, or

$$
\left|F^{\prime}(z)\right|^{2} \neq 0, \quad \zeta \leq 0
$$




\subsubsection{Example}

As an example, let $\rho(\phi)=1-.2 \cos (2 \phi)+.1 \sin (3 \phi)+.1 \cos (7 \phi) . \delta_{j}$, and $\epsilon_{j}$ were calculated for values of $j=40$. Figure(2.3a) shows lines of $u=$ constant, with the innermost curve being $u=0$, along with the parameterization in cylindrical coordinates. As $u$ increases, the coordinates are becoming circular. Figure $(2.3 \mathrm{~b})$ shows the surfaces $\zeta=$ constant, with the outermost curve being $\zeta=0$, again with the original curve in cylindrical coordinates. As $\zeta$ decreases, the coordinates are becoming circular. Because the coordinates are becoming circular far away from the boundary, there will be no need to perform an inverse mapping. As can be seen from this example, there is excellent agreement between a curve given in cylindrical coordinates and one on which $u=$ constant using the algorithm given above.

\subsection{Solutions of the Helmholtz Equation}

As mentioned previously, changing variables conformally changes the Helmholtz equation. The method by which the new Helmholtz equation is solved is described in this section. The method is broadly similar for the fields outside and inside the scatterer. The details of both derivations are developed in the following subsections.

\subsubsection{Exterior}

After conformally mapping the exterior of the scatterer to an upper halfspace, the Helmholtz equation is transformed to the following:

$$
P_{u u}+P_{v v}+k^{2} \mathcal{G}(u, v) P=0
$$


a.

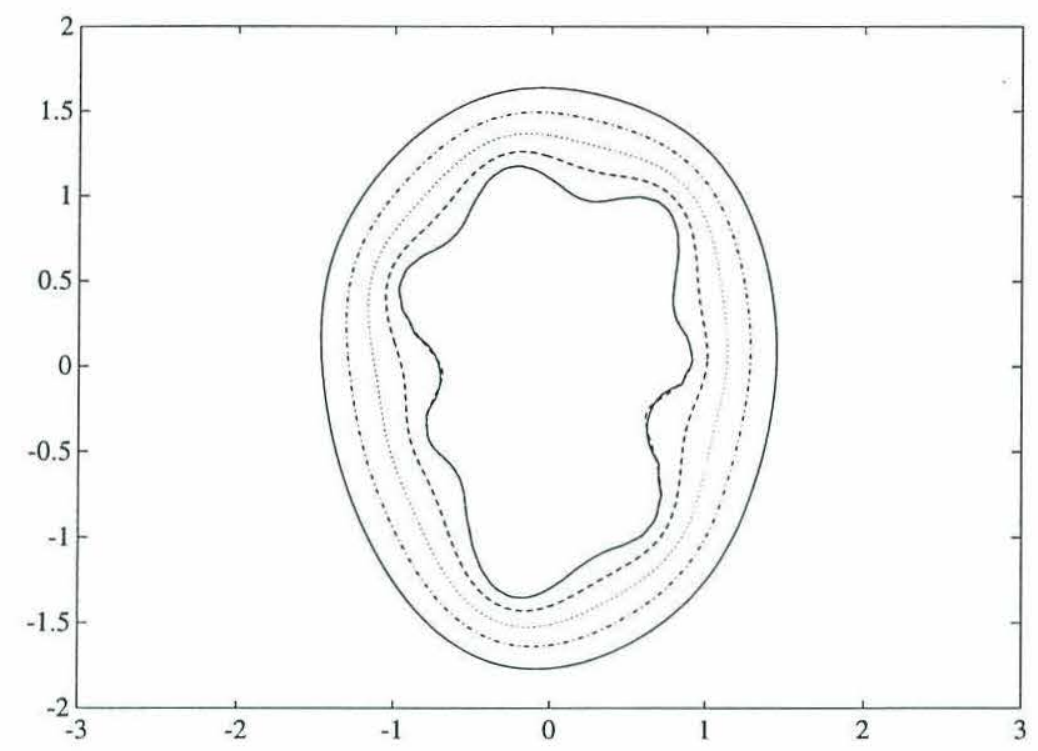

b.

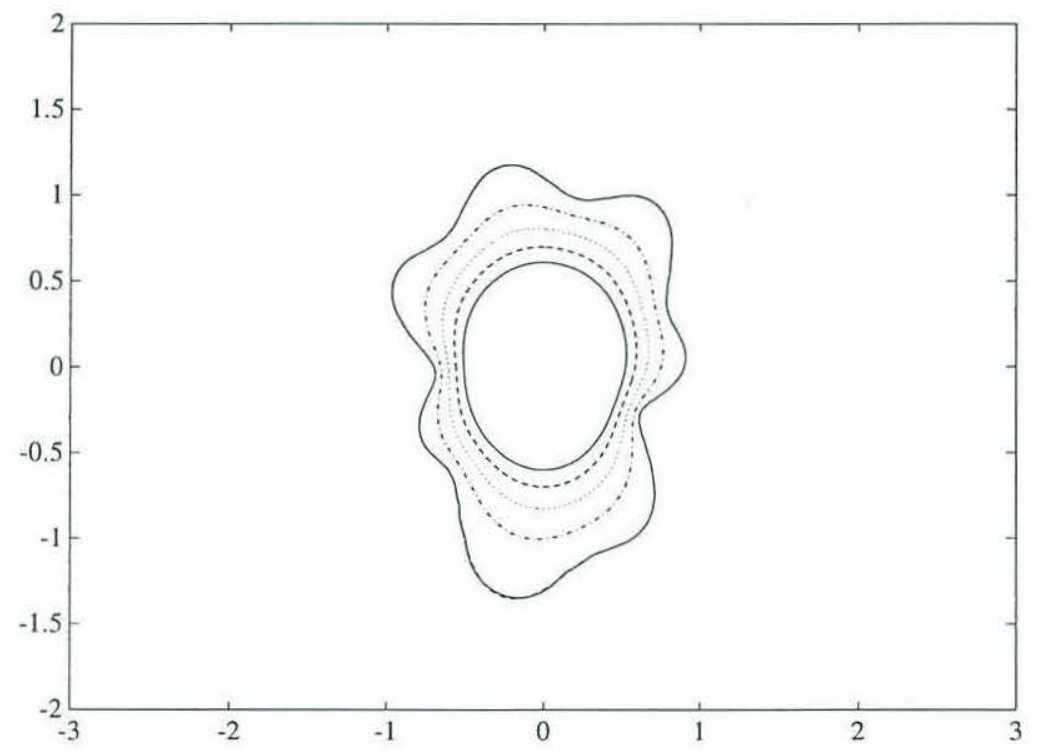

Figure 2.3: Example of interior and exterior mapping: (a) curves $u=$ constant for several values of $u$, with the inner curve showing $u=0$ along with the original parameterization in circular coordinates. (b) curves $\zeta=$ constant for several values of $u$, with the outer curve showing $\zeta=0$ along with the original parameterization in circular coordinates. 
where $\mathcal{G}(u, v)$ is a function which depends on the specific transformation. More specifically, it is the Jacobian of the transformation, or

$$
\mathcal{G}(u, v)=x_{u}^{2}+x_{v}^{2}
$$

where $x_{u}$ and $x_{v}$ are the partial derivatives of $x(u, v)$ with respect to $u$ and $v$. Note that the uniqueness condition for the map was that the Jacobian be non-zero. If the Jacobian were zero, this would correspond to a zero wavenumber, or an infinite soundspeed, which is non-physical.

The solutions of this Helmholtz equation are in the form

$$
e^{i k[x \cos (\mu)+y \sin (\mu)]}
$$

where $\mu$ is a parameter. Physically, this solution represents a wave propagating at an angle $\mu$ with respect to the $x$ axis. By integrating over all possible values of $\mu$, the solution may be written as

$$
P=\int_{W_{0}} A(\mu) e^{i k[x \cos (\mu)+y \sin (\mu)]} d \mu+\int_{W_{1}} B(\mu) e^{i k[x \cos (\mu)+y \sin (\mu)]} d \mu
$$

where $W_{0}$ and $W_{1}$ are contours chosen such that the integrals will represent the incident and scattered waves, respectively, and $A(\mu)$ and $B(\mu)$ will be the amplitudes of the incident and scattered waves,respectively, propagating at angle $\mu$. In general $A(\mu)$ is known, and $B(\mu)$ is a function that must be solved for. Since $A(\mu)$ and $B(\mu)$ must be periodic functions, they may be expanded as Fourier series:

$$
A(\mu)=\frac{1}{2 \pi} \sum_{n=-\infty}^{\infty} a_{n} e^{i n \mu}, \quad B(\mu)=\frac{1}{2 \pi} \sum_{n=-\infty}^{\infty} b_{n} e^{i n \mu} .
$$

Therefore, the values of $a_{n}$ are known, and the values of $b_{n}$ are unknown constants describing the scattered field.

There are various ways to evaluate the above integrals, each with different numerical properties. The easiest is to collect terms in the exponential as follows:

$$
\begin{gathered}
r(u, v)=\sqrt{x^{2}+y^{2}} \\
\theta(u, v) \arctan \left(\frac{y}{x}\right) .
\end{gathered}
$$


Inserting Eqs.(2.24 and 2.25)into Eq.(2.23), yields

$$
P=\int_{W_{0}} \frac{1}{2 \pi} \sum_{n=-\infty}^{\infty} a_{n} e^{i n \mu} e^{i k r(u, v) \cos (\theta(u, v)-\mu)} d \mu+\int_{W_{1}} \frac{1}{2 \pi} \sum_{n=-\infty}^{\infty} b_{n} e^{i n \mu} e^{i k r(u, v) \cos (\theta(u, v)-\mu)} d \mu .
$$

It is here where the contours necesary for $W_{0}$ and $W_{1}$ become apparent. This is the same integral that arises in the solution of the wave equation in circular coordinates. According to Sommerfeld [32], by choosing these contours, the above integral may be evaluated analytically, leading to

$$
P=\sum_{n=-\infty}^{\infty} a_{n} i^{n} e^{i n \theta(u, v)} J_{n}(k r(u, v))+\sum_{n=-\infty}^{\infty} b_{n} i^{n} e^{i n \theta(u, v)} H_{n}^{(1)}(k r(u, v))
$$

Where $J_{n}$ and $H_{n}^{(1)}$ are Bessel and Hankel functions of the first kind, respectively, of order $n$. Several things may be seen immediately from this expression. If the scatterer is circular, $\theta(u, v)=v$, and $r(u, v)=g_{-1} e^{u}$, and the solution is precisely the solution obtained from separation of variables in circular coordinates. For the noncircular case, as $u \rightarrow \infty, \theta(u, v) \rightarrow v$, and $r(u, v) \rightarrow g_{-1} e^{u}$, so the solution will approach the circular solution (i.e. a summation of Bessel and Hankel functions, although the constants $b_{n}$ will be different) far from the scatterer. The first term in this expression is the incident field, and the second is the scattered field. By assuming a unit amplitude plane wave excitation, with the plane wave coming from an angle $v_{i}$ with respect to the $x$-axis, the total field is $P=P^{i n c}+P^{\text {scat }}$, where $P^{i n c}$ is the incident field, and $P^{\text {scat }}$ is the scattered field.

$$
\begin{aligned}
P^{i n c} & =\sum_{n=-\infty}^{\infty} a_{n} i^{n} e^{i n \theta(u, v)} J_{n}(k r(u, v))=e^{i k\left[x \cos \left(v_{i}\right)+y \sin \left(v_{i}\right)\right]} \\
P^{\text {scat }} & =\sum_{n=-\infty}^{\infty} b_{n} i^{n} e^{i n \theta(u, v)} H_{n}^{(1)}(k r(u, v))
\end{aligned}
$$

As stated above, far away from the scatterer the coordinates become circular, so using this fact and the asymptotic form of the Hankel functions, the farfield scattered 


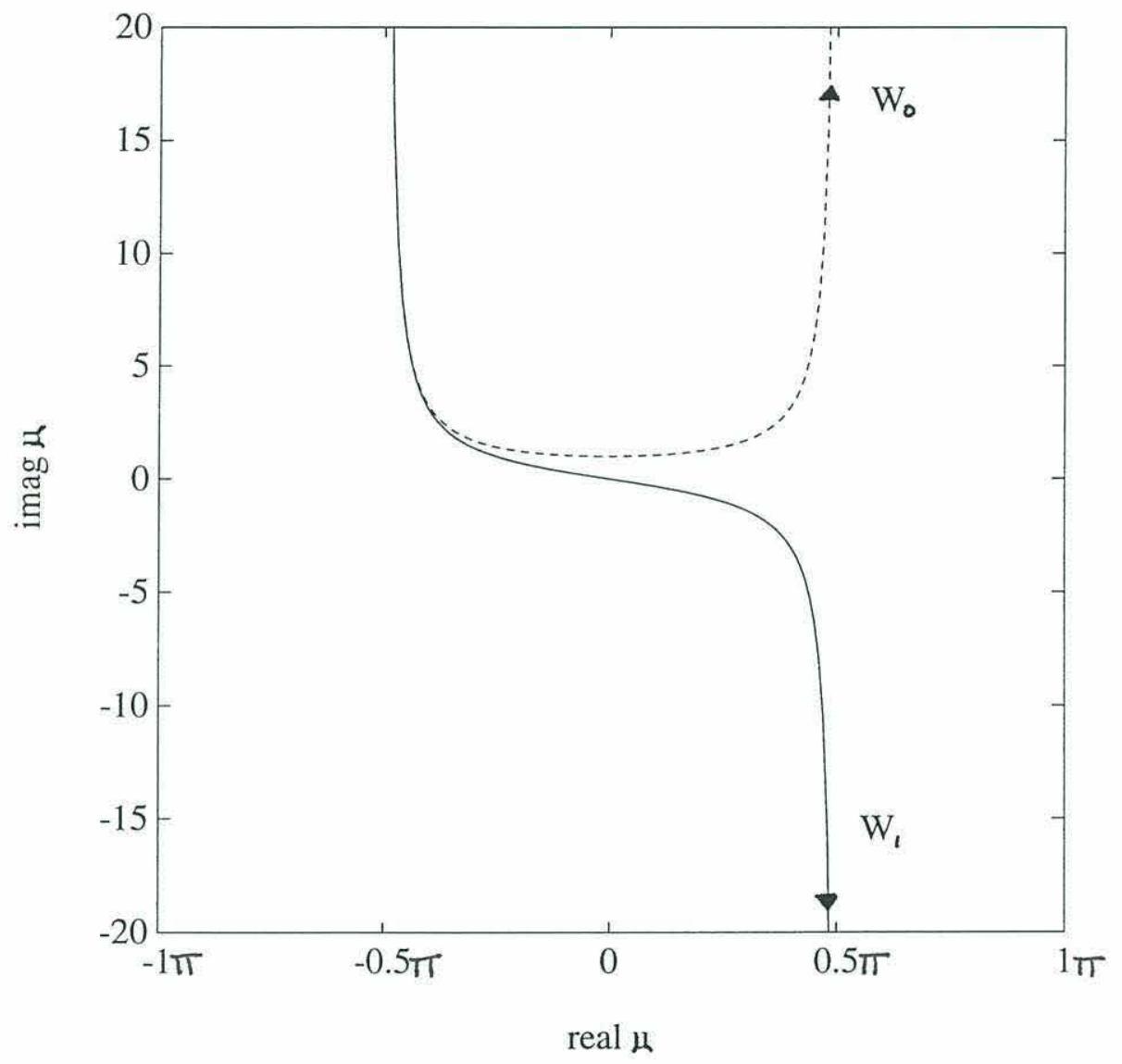

Figure 2.4: Contours of Integration 
pressure is given by

$$
P_{u \rightarrow \infty}^{s c a t}=\frac{e^{i k g_{-1} e^{u}}}{\sqrt{g_{-1} e^{u}}} \underbrace{\sqrt{\frac{2}{\pi k}} e^{-i \frac{\pi}{4}} \sum_{n=-\infty}^{\infty} b_{n} e^{i n v}}_{f^{\infty}(v)}
$$

where $f^{\infty}(v)$ is the form function[33]. This is the quantity that will be generated in the results section.

\subsubsection{Interior}

The problem of determining the field inside the cylinder proceeds in almost exactly the same manner as for the exterior field. Since there is a different mapping for the interior, there is also have a different Helmholtz equation:

$$
P_{\zeta \zeta}+P_{\eta \eta}+k_{1}^{2} \mathcal{F}(\zeta, \eta) P=0
$$

where $k_{1}$ is the wavenumber inside the scatterer, and $\mathcal{F}(\zeta, \eta)$ is the Jacobian of the inner mapping. Following the same procedure as for the exterior case, the interior pressure is

$$
P=\int_{W_{0}} C(\mu) e^{i k_{1}[x \cos (\mu)+y \sin (\mu)]} d \mu+\int_{W_{1}} D(\mu) e^{i k_{1}[x \cos (\mu)+y \sin (\mu)]} d \mu,
$$

where $C(\mu)$ and $D(\mu)$ are unknown fields. Expanding each of these as Fourier series and integrating leads to

$$
P=\sum_{n=-\infty}^{\infty} c_{n} i^{n} e^{i n \theta(\zeta, \eta)} J_{n}\left(k_{1} r(\zeta, \eta)\right)+\sum_{n=-\infty}^{\infty} d_{n} i^{n} e^{i n \theta(\zeta, \eta)} H_{n}^{(1)}\left(k_{1} r(\zeta, \eta)\right)
$$

and

$$
\begin{aligned}
& r(\zeta, \eta)=\sqrt{x^{2}+y^{2}} \\
& \theta(\zeta, \eta) \arctan \left(\frac{y}{x}\right)
\end{aligned}
$$

with $x$ and $y$ expressed in terms of $\zeta$ and $\eta$. From the form of the interior mapping, as $\zeta \rightarrow-\infty, r(\zeta, \eta) \rightarrow 0$. However, the Hankel functions become infinite as its argument 
approaches zero. Since the field must be finite everywhere, $d_{n} \equiv 0$. This leaves for the interior pressure

$$
P^{i n t}=\sum_{n=-\infty}^{\infty} c_{n} i^{n} e^{i n \theta(\zeta, \eta)} J_{n}\left(k_{1} r(\zeta, \eta)\right)
$$

The integral which led to the above expression for the interior pressure is:

$$
P^{i n t}=\int_{W_{0}} \sum_{n=-\infty}^{\infty} c_{n} e^{i n \mu} e^{i k_{1}[x \cos (\mu)+y \sin (\mu)]} d \mu .
$$

Since all that is required is this function on the boundary, let $\left[x\left(\zeta_{0}, \eta\right), y\left(\zeta_{0}, \eta\right)\right]=$ $\left[x\left(u_{0}, v\right), y\left(u_{0}, v\right)\right]$. Therefore, the interior pressure may be rewritten as a function of the exterior variable $u, v$ :

$$
P^{i n t}=\sum_{n=-\infty}^{\infty} c_{n} i^{n} e^{i n \theta_{s}} J_{n}\left(k_{1} \rho_{s}\right)
$$

where $\rho_{s}=r\left(u_{0}, v\right), \theta_{s}=\theta\left(u_{0}, v\right)$. To summarize, in this section expressions were developed for the pressure fields inside and outside the scatterer. These are expressed in terms of circular eigenfunctions, but now they are functions of the new angular variable $v$. In Chapter 3 this fact will be exploited in developing a new method for satisfying the boundary conditions.

\subsection{Boundary conditions}

The boundary conditions that must be satisfied on the scatterer surface are continuity of pressure and normal particle velocity. In the remainder of this section all quantities discussed will be at the boundary. Expressions for the pressure were derived above. The normal particle velocity $V_{n}$ is proportional to the normal derivative of the pressure $P_{n}$, or

$$
V_{n}=\frac{P_{n}}{i \omega \rho}
$$

This requires the calculation of the normal derivative of the pressure. Due to the fact that the change of variables was conformal, and the surface is $u=u_{o}$, the normal derivative 
is easy to calculate. The normal derivative is $P_{n}=\hat{n} \cdot \nabla P$, where $\hat{n}=\hat{a_{u}}$ is a unit vector in the $u$ direction. From [34], it is known that

$$
\nabla P=\frac{\hat{a_{u}}}{h_{u}} \frac{\partial P}{\partial u}+\frac{\hat{a_{v}}}{h_{v}} \frac{\partial P}{\partial v}
$$

where $\hat{a_{v}}$ is a unit vector in the $v$ direction, and $h_{u}$ and $h_{v}$ are scale factors of the transformation and are given by

$$
\begin{aligned}
h_{u} & =\sqrt{x_{u}^{2}+y_{u}^{2}} \\
h_{v} & =\sqrt{x_{v}^{2}+y_{v}^{2}} .
\end{aligned}
$$

Therefore, the normal particle velocity is $V_{n}=\frac{P_{u}}{i \omega \rho h_{u}}$. Expressing the integrals in terms of $u$ and $v$ also facilitates the computation of $P_{u}$. Consider the integral representation of the typical pressure field (this is for the scattered field, but the procedure is the same for the incident and the interior field):

$$
P=\frac{1}{2 \pi} \int_{W_{1}} \sum_{m=-\infty}^{\infty} b_{m} e^{i m \mu} e^{i k[x \cos (\mu)+y \sin (\mu)]} d \mu
$$

Differentiating with respect to $u$ gives

$$
P_{u}=\frac{1}{2 \pi} \int_{W_{1}} \sum_{m=-\infty}^{\infty} b_{m} e^{i m \mu} i k\left(x_{u} \cos (\mu)+y_{u} \sin (\mu)\right) e^{i k[x \cos (\mu)+y \sin (\mu)]} d \mu .
$$

Rearranging and performing the integrations yields

$$
P_{u}=\sum_{m=-\infty}^{\infty} b_{m}\left[D^{-} e^{i(m-1)\left(\theta_{s}+\frac{\pi}{2}\right)} H_{m-1}^{(1)}\left(k r_{s}\right)-D^{+} e^{i(m+1)\left(\theta_{s}+\frac{\pi}{2}\right)} H_{m+1}^{(1)}\left(k r_{s}\right)\right]
$$

where

$$
\begin{aligned}
D^{-} & =\frac{1}{2}\left(x_{u}\left(u_{0}, v\right)+i y_{u}\left(u_{0}, v\right)\right) \\
D^{+} & =\frac{1}{2}\left(x_{u}\left(u_{0}, v\right)-i y_{u}\left(u_{0}, v\right)\right) .
\end{aligned}
$$

So now expressions have been derived for all the quantities necesary to satisfy the boundary conditions, namely continuity of pressure and normal particle velocity. The next chapter deals with the numerical issues that arise when actually trying to satisfy the boundary conditions. 


\section{Chapter 3}

\section{Numerical Considerations}

Some of the numerical issues that arise in the solution of this problem are treated in this chapter. Among these are how the boundary conditions are satisfied, a convergence criterion, and how to calculate the basis functions efficiently.

\subsection{Boundary Conditions}

The most difficult part of the scattering problem under consideration is how to satisfy the boundary conditions. Although the expressions that were derived for the scattered field on the surface appear to be similar to that of the circular cylinder, there is one major difference: the modal functions are not orthogonal. This makes it much more difficult to choose the scatered field coefficients properly. In the following section the problem will be explained better, and it will be shown how to overcome it.

For simplicity, the impenetrable case will be discussed, but the issue is the same for the penetrable case. The expressions for the fields derived in the previous chapter are expanded in terms of basis functions which are solutions of the Helmholtz equation. For example, from Eq. $(2.28)$ the basis functions $P_{m}(v)$ for the scattered pressure on the 
surface are

$$
P_{m}(v)=i^{m} e^{i m \theta\left(u_{0}, v\right)} H_{m}^{(1)}\left(k r\left(u_{0}, v\right)\right) .
$$

When treating rigid boundary conditions, the following basis functions from Eq.(2.37 and 2.42) will be used:

$$
V_{m}^{s c a t}(v)=\frac{1}{i \omega \rho h_{u}} \frac{\partial P_{m}}{\partial u}
$$

An infinite summation of these basis functions should be able to match the boundary condition. In practice however, infinite summations cannot be performed, and therefore the summations must be truncated at some point. This is a major obstacle, because it is far from obvious where to truncate this summation. For the separable geometries the modal functions are eigenfunctions of a Sturm-Liouville problem, and therefore are orthogonal. Multiplying the boundary condition equation by one of the eigenfunctions and integrating over the angular variable will thus leave a closed form expression for the unknown constant. Therefore, truncating the summations for a separable geometry will not affect the accuracy of the constants that are solved for.

For a nonseparable geometry, the modal functions are no longer orthogonal. Because of this fact, questions arise on which functions are appropriate by which to multiply the boundary condition (since there are no eigenfunctions). No matter what set of functions are chosen, the result of multiplying by the weighting functions and integrating will be a system of equations to be solved for the unknown coefficients. It is here where the truncation problem arises. Since all of the coefficients are coupled to each other, truncation of the series will affect the accuracy of the constants that are solved for.

The following example will demonstrate how this non-orthogonality of modal functions can make the boundary conditions far more difficult to satisfy. 


\subsubsection{Example: Circular cylinder}

Before treating the noncircular cylinder, a case will be examined for which an exact solution exists, the circular cylinder. In this case, separation of variables shows that the basis functions on the surface of the scatterer are

$$
V_{m}^{s c a t}(v)=\frac{1}{i \omega \rho h_{u}} b_{m} i^{m} e^{i m v} H_{m}^{\prime(1)}\left(k g_{-1} e^{u_{0}}\right) k g_{-1} e^{u_{0}}
$$

This is the standard expression since for a circular coordinate system $v=\theta$ and $g_{-1} e^{u_{0}}=$ $a$, where $a$ is the radius of the scatterer. Here the basis functions are proportional to $e^{i m v}$, which constitute an orthogonal set, and therefore any incident field may be represented in terms of this set of basis functions. The truncation properties of this problem are well known. The series may be truncated so that $|m| \leq M$, where $M$ is somewhat larger than $k e^{u_{0}}$. This truncation point is arrived at because the incident field is expanded in terms of $J_{m}\left(k e^{u_{0}}\right)$. Once $M>k e^{u_{0}}$, the value of the bessel function becomes small, and therefore the amount of energy contained in this mode is negligible. Therefore, in terms of a finite summation, the boundary condition becomes

$$
\sum_{m=-M}^{M} b_{m} V_{m}^{\text {scat }}(v)+V^{\text {inc }}(v)=0,
$$

where $V^{i n c}(v)$ is the known incident particle velocity on the boundary and the $b_{m}$ are the scattered field coefficients that must be chosen. This would be solved by multiplying by $e^{-i m v}$ for $|m| \leq M$ and integrating over the range of $v$ to get

$$
b_{m}=-a_{m} \frac{J_{m}^{\prime}\left(k e^{u_{0}}\right)}{H_{m}^{\prime(1)}\left(k e^{u_{0}}\right)}
$$

where $a_{m}=e^{-i m \theta_{0}}$ and $\theta_{0}$ is the angle of incidence of the incident plane wave.

Now suppose that the coefficients $b_{m}$ are a linear combination of some new set of coefficients $b_{n}^{(1)}$

$$
b_{m}=\sum_{n=-N}^{N} Q_{m, n} b_{n}^{(1)}
$$


where $Q$ is some known matrix. Inserting this into Eq.(3.4) and rearranging the summations, leaves

$$
V^{i n c}=-\sum_{n=-N}^{N} b_{n}^{(1)} \underbrace{\sum_{m=-M}^{M} Q_{m, n} V_{m}^{\text {scat }}(v)}_{\Phi_{n}^{\text {scat }}(v)} .
$$

This has the effect of expanding the solution in terms of a new set of basis functions $\Phi_{n}^{s c a t}(v)$. Note that these functions are no longer orthogonal, although they are each still a solution of the Helmholtz equation, because each $\Phi_{n}^{s c a t}(v)$ is a linear combination of the $V_{m}^{s c a t}(v)$.

Further assume that $N$ is less than $M$. Under this assumption, trying to satisfy the boundary conditions in the same manner as before results in an overdetermined problem. Multiplying by $e^{-i m v}$ for $|m| \leq M$ and integrating gives $M$ constraints, and only $N$ unknowns. This may be solved by minimizing the mean squared error, but nonetheless, error will be introduced. This method will be referred to as Method 1 .

Another alternative is to multiply by $e^{-i m v}$ for $|m| \leq N$, which would yield an exactly determined problem, but now there is no constraint on the $e^{i m v}$ for $|N|<m \leq|M|$ so again there will be error. This will be referred to as Method 2. The conclusion therefore is that in either case there will be some error introduced, although the error is not the same in each case. In Method 1 the error will be distributed throughout all of the angular frequency components $e^{i m v}$, whereas in Method 2 all the error will be at the higher frequencies.

From this example it is seen that by choosing the incorrect set of basis functions a problem that can be solved exactly can be turned into one that will necesarily contain some error.

It should not be a surprise that a problem that can be solved exactly can be turned into a more difficut one. In this case, choosing a specific $Q\left(Q_{n, m}=J_{n-m}\left(k r_{1}\right) e^{i(n-m) v_{1}}\right)$, physically represents moving the coordinate origin from the center of the circle to $x_{c}=$ 
$r_{1} \cos \left(v_{1}\right), y_{c}=r_{1} \sin \left(v_{1}\right)$. The result of this is a radius that changes as a function of angle, which is the same as the noncircular case. Therefore, it is expected that the resulting equations should be similar to that of the noncircular case.

\subsubsection{Noncircular cylinder}

The same problem of non-orthogonality of modal functions arises in conjunction with the noncircular cross section problem. There is an infinite summation of nonorthogonal basis functions, which must be truncated somewhere. However, once the series is truncated error will be introduced when choosing the unknown coefficients, presumably because an inappropriate set of basis functions was used. Ideally, there would be some way to construct the appropriate functions from the basis functions derived previously in conjunction with the transformed Helmholtz equation, but this is a very difficult problem. Since it is not known a priori what the appropriate basis functions are, a method must be developed to choose the unknown coefficients appropriately.

Both methods 1 and 2 described above are in fact special cases of the same method. Recall the boundary condition after truncating the series for the scattered field is

$$
\sum_{m=-M}^{M} b_{m} V_{m}^{s c a t}(v)+V^{i n c}(v)=0
$$

Multiplying by a set of functions $\psi_{n}(v)$ and integrating over the range of $v$ yields

$$
\sum_{m=-M}^{M} b_{m} \int_{0}^{2 \pi} V_{m}^{s c a t}(v) \psi_{n}(v) d v+\int_{0}^{2 \pi} V^{i n c}(v) \psi_{n}(v) d v=0
$$

for each $n$. The question now is to choose an appropriate set of $\psi$. A logical choice is to try to minimize the mean squared error $\Omega$ on the surface, or

$$
\Omega=\left\|\sum_{m=-M}^{M} b_{m} V_{m}^{s c a t}(v)+V^{i n c}(v)\right\|
$$


where

$$
\left\|\sum_{m=-M}^{M} b_{m} V_{m}^{\text {scat }}(v)+V^{i n c}(v)\right\|=\left[\int_{0}^{2 \pi}\left|\sum_{m=-M}^{M} b_{m} V_{m}^{\text {scat }}(v)+V^{i n c}(v)\right|^{2} d v\right]^{1 / 2}
$$

In order to minimize this expression, differentiate with respect to $b_{n}$ and set equal to zero to obtain

$$
\int_{0}^{2 \pi} \sum_{m=-M}^{M} b_{m} V_{m}^{\text {scat }}(v) V_{n}^{\text {scat* }}(v) d v+\int_{0}^{2 \pi} V^{\text {inc }}(v) V_{n}^{\text {scat } *}(v) d v=0
$$

for $n \leq M$. Comparing Eq.(3.12) to Eq.(3.9), it is seen that this is the same as choosing $\psi_{n}=V_{n}^{\text {scat* }}(v)$. This choice of weighting function is equivalent to the mode matching method (MMM)[31]. There is a proof that this will converge to the true solution with increasing $M$, but in practice, $M$ will be very large.

Another choice for $\psi$ is to let $\psi_{n}=e^{i n v}$. This choice of weighting function is referred to as the Fourier matching method (FMM). It is here where the conformal mapping becomes important. Recall that this $v$ is specific to the geometry. It was generated through the conformal mapping algorithm. Without changing variables to $(u, v)$, this choice of $\psi$ would not be possible. The author cannot prove rigorously that this will converge to the true solution. However, as can be seen in Chapter 4, in all of the cases that have been tried, the farfield scattered pressure calculated using the FMM compares extremely well with either the exact solution (for the elliptic cylinder), the high frequency limit predicted using the physical optics approximation (for the impenetetrable ellipse and three-leaf clover), or the DWBA (for the weakly scattering square). The FMM is in general preferable to the MMM for calculating the farfield scattered pressure because far fewer terms are required for a convergent solution. For example, for a 2:1 aspect ratio rigid elliptic cylinder using the $\mathrm{MMM}, M=50$ is required for a convergent solution at $k a=3$. For the same problem using the FMM, there is a converged solution for $M=12$. However, if a nearfield solution is desired, the MMM must be used, since the error in satisfying the boundary condition using the FMM does not approach zero with increasing $M$. 
In summary, the equations for the boundary conditions using the FMM are for rigid boundary conditions:

$$
\int_{0}^{2 \pi} \sum_{m=-M}^{M} b_{m} V_{m}^{s c a t}(v) e^{-i n v} d v+\int_{0}^{2 \pi} V^{i n c}(v) e^{-i n v} d v=0,
$$

for soft boundary conditions,

$$
\int_{0}^{2 \pi} \sum_{m=-M}^{M} b_{m} P_{m}^{s c a t}(v) e^{-i n v} d v+\int_{0}^{2 \pi} P^{i n c}(v) e^{-i n v} d v=0,
$$

and for penetrable fluid boundary conditions,

$$
\begin{array}{r}
\int_{0}^{2 \pi} \sum_{m=-M}^{M} b_{m} P_{m}^{s c a t}(v) e^{-i n v} d v+\int_{0}^{2 \pi} P^{i n c}(v) e^{-i n v} d v=\int_{0}^{2 \pi} \sum_{m=-M}^{M} c_{m} P_{m}^{i n t}(v) e^{-i n v} d v \\
\int_{0}^{2 \pi} \sum_{m=-M}^{M} b_{m} V_{m}^{s c a t}(v) e^{-i n v} d v+\int_{0}^{2 \pi} V^{i n c}(v) e^{-i n v} d v=\frac{\rho_{1} c_{1}}{\rho c} \int_{0}^{2 \pi} \sum_{m=-M}^{M} c_{m} V_{m}^{i n t}(v) e^{-i n v} d v
\end{array}
$$

for $n \leq M$.

\subsection{Convergence}

Since for the FMM, the error on the boundary does not necesarily decrease with increasing $M$, another criterion for convergence is used. Therefore, a criterion using the field quantities away from the scatterer surface will be used. The following is a measure

of how well the optical theorem is satisfied [31], and is used in the MMM. Since the MMM and the FMM have similar forms, it may be used directly term by term as given in $[31]$ :

$$
\epsilon(M)=2 \frac{\left.\left|\sum_{m=-M}^{M}\right| b_{m}\right|^{2}+R e \sum_{m=-M}^{M} b_{m} e^{i m\left(\theta+\frac{\pi}{2}\right)} \mid}{\left.\left|\sum_{m=-M}^{M}\right| b_{m}\right|^{2}-\operatorname{Re} \sum_{m=-M}^{M} b_{m} e^{i m\left(\theta+\frac{\pi}{2}\right)} \mid},
$$

where $\epsilon(M)$ is the error as a function of the number of unknown coefficients. The numerator is a measure of how well the optical theorem is satisfied. The first term in the 
numerator is simply the total cross section $\sigma$, which is the total scattered power normalized by the incident power density. The denominator is approximately twice the total cross section $\sigma$ and therefore normalizes the error in the optical theorem by the total scattered power.

In practice, $\mathrm{M}$ will be increased until $\epsilon(M)$ is smaller than some given tolerance. This is a necesary condition that the field must satisfy (i.e. this is a statement of conservation of energy, so it must be satisfied), but it is not sufficient.

The insufficiency of this criterion occurs because there are many choices for $b_{m}$ that satisfy the above condition. If the $b_{m}$ were to be chosen simply to satisfy this condition, the choice would not be unique. The above condition is totally independent of the type of scatterer, both shape and whether the boundary condition is soft, rigid or penetrable.

The farfield condition becomes useful only when used in conjunction with the conditions imposed by the FMM on the scatterer surface. As stated previously, the FMM only matches the boundary condition to some order of $e^{i m v}$. It is unclear how to choose $M$. The above criterion gives us a measure of how large $M$ should be. For example, the boundary condition may be met in the FMM sense for some $M$. If that $M$ is not large enough, $\epsilon_{M}$ may not be small, and therefore that choice of $b_{m}$ will not satisfy a necesary condition. By increasing $M$ until $\epsilon_{M}$ is less than some tolerance. Therefore, out of all the possible choices of $b_{m}$ that satisfy the above criterion, we choose the ones that also satisfy the boundary conditions in the FMM sense.

In all cases that have been tried, when $\epsilon(M)$ decreases to within some suitably small tolerance, the backscattered pressure is no longer changing significantly, and compares well with the reference solutions. Specific examples are given in Chapter 4. 


\subsection{Numerical Computation of Modal Functions}

In Chapter 2, expressions were derived for the pressure and velocity fields in terms of series of Bessel functions. When trying to satisfy the boundary conditions, $2 M+1$ Bessel functions must be calculated for $\mathrm{N}$ integration points. This can be numerically intensive. The following gives an improved method for calculating the mode functions. Recalling the integral representation of the scattered field and rearranging somewhat leaves

$$
P^{s c a t}=\int_{W_{1}} \sum_{n=-\infty}^{\infty} b_{n} e^{i n \mu} e^{i k g_{-1} e^{u} \cos (v-\mu)} K(u, v, \mu) d \mu,
$$

where

$$
K(u, v, \mu)=e^{i k[x \cos (\mu)+y \sin (\mu)]} e^{-i k g_{-1} e^{u} \cos (v-\mu)} .
$$

Since $K(u, v, \mu)$ is a periodic function in $\mu$, it may be expanded in a Fourier series as follows:

$$
K(u, v, \mu)=\frac{1}{2 \pi} \sum_{n=-\infty}^{\infty} \kappa_{n} e^{i n \mu},
$$

where $\kappa_{n}$ is a function of $u$ and $v$, and may be found by integrating as follows

$$
\kappa_{n}=\int_{0}^{2 \pi} e^{-i n \mu} K(u, v, \mu) d \mu .
$$

The $\kappa_{n}$ are known analytically, but in general the result is a multiple summation of products of Bessel functions, and the truncation of these series is far from obvious. It is also far more efficient to perform the above integration numerically, and since that integration is essentially a Fourier transform, a Fast Fourier Transform (FFT) algorithm may be used to calculate it. For simple cases, where there is only one roughness coefficient, it may be easier to find $\kappa_{n}$ analytically. For example, for an ellipse, $g_{1}$ is the only nonzero coefficient, and

$$
\kappa_{n}=J_{n}\left(k g_{1} e^{-u}\right) i^{n} e^{i n v} .
$$

In general, the resulting integral becomes

$$
P^{s c a t}=\int_{W_{1}}\left[\sum_{n=-\infty}^{\infty} b_{n} e^{i n \mu}\right]\left[\sum_{n=-\infty}^{\infty} e^{i n \mu} \kappa_{n}\right] e^{i k g-1 e^{u} \cos (v-\mu)} d \mu .
$$


Multiplying these series together gives

$$
P^{s c a t}=\sum_{n=-\infty}^{\infty} \sum_{l=-\infty}^{\infty} \kappa_{l} \int_{W_{1}} b_{n-l} e^{i n \mu} e^{i k g-1} e^{u} \cos (v-\mu) d \mu .
$$

This may be evaluated analytically, and after rearranging the summations,

$$
P^{s c a t}=\sum_{m=-\infty}^{\infty} b_{m} \sum_{n=-\infty}^{\infty} \kappa_{n-m} i^{n} e^{i n v} H_{n}^{(1)}\left(k g_{-1} e^{u}\right) \text {. }
$$

Although this appears more complicated than the previous expression (Eq.(2.28)), it is more efficient to evaluate because each $H_{n}^{(1)}$ needs to be evaluated only once for a given frequency, instead of $\mathrm{N}$ times for the previous expression.

A similar expression may be derived for the normal particle velocity. Due to the conformal nature of the change of variables, $\frac{\partial}{\partial n} \propto \frac{\partial}{\partial u}$. Therefore the normal particle velocity is $V^{\text {scat }}=\frac{1}{\omega \rho h_{u}} P_{u}^{s c a t}$. Performing this differentiation yields

$$
V^{s c a t}=\frac{1}{i \omega \rho h_{u}} \int_{W_{1}} \sum_{n=-\infty}^{\infty} b_{n} e^{i n \mu} e^{i k g_{-1} e^{u} \cos (v-\mu)} K(u, v, \mu)\left[x_{u} \cos (\mu)+y_{u} \sin (\mu)\right] d \mu .
$$

By letting

$$
K^{\prime}(u, v, \mu)=K(u, v, \mu)\left[x_{u} \cos (\mu)+y_{u} \sin (\mu)\right]
$$

$K^{\prime}$ can be expanded as a Fourier series:

$$
K^{\prime}(u, v, \mu)=\sum_{n=-\infty}^{\infty} \kappa_{n}^{\prime} e^{i n \mu}
$$

Following the same procedure as for the pressure the normal particle velocity is given by:

$$
V^{\text {scat }}=\frac{1}{\omega \rho h_{u}} \sum_{m=-\infty}^{\infty} b_{m} \sum_{n=-\infty}^{\infty} \kappa_{n-m}^{\prime} i^{n} e^{i n v} H_{n}^{(1)}\left(k g_{-1} e^{u}\right)
$$

The normal particle velocity of the incident field is known immediately from Eq.(2.37):

$$
V^{i n c}=\frac{1}{\omega \rho h_{u}} e^{i k\left[x \cos \left(v_{i}\right)+y \sin \left(v_{i}\right)\right]}\left[x_{u} \cos \left(v_{i}\right)+y_{u} \sin \left(v_{i}\right)\right] .
$$

The integral which led to the above expression for the interior pressure is

$$
P^{i n t}=\int_{W_{0}} \sum_{n=-\infty}^{\infty} c_{n} e^{i n \mu} e^{i k_{1}[x \cos (\mu)+y \sin (\mu)]} d \mu .
$$


Following the same procedure as for the exterior problem, gives

$$
P^{i n t}=\int_{W_{1}} \sum_{n=-\infty}^{\infty} c_{n} e^{i n \mu} T(u, v, \mu) d \mu,
$$

where

$$
T(u, v, \mu)=e^{i k_{1}[x \cos (\mu)+y \sin (\mu)]} .
$$

Since $T(u, v, \mu)$ is a periodic function in $\mu$, it may be expanded in a Fourier series as follows:

$$
T(u, v, \mu)=\frac{1}{2 \pi} \sum_{n=-\infty}^{\infty} \tau_{n} e^{i n \mu}
$$

where $\tau_{n}$ is a function of $u$ and $v$, and may be found by integrating as follows

$$
\tau_{n}=\int_{0}^{2 \pi} e^{-i n \mu} T(u, v, \mu) d \mu
$$

Inserting Eq.(3.34) into Eq.(3.31) in and multiplying the series yields

$$
P^{i n t}=\sum_{n=-\infty}^{\infty} \sum_{m=-\infty}^{\infty} c_{n-m} \tau_{m} \int_{W_{0}} e^{i n \mu} d \mu .
$$

This integral is zero unless $n=0$, so letting $n=0$ and rearranging the sum, the internal pressure becomes

$$
P^{i n t}=\sum_{m=-\infty}^{\infty} c_{m} \tau_{-m}
$$

The normal particle velocity may also be found very easily. Taking the normal derivative of the internal pressure with respect to the normal gives:

$$
V^{\text {int }}=\frac{1}{\omega \rho h_{u}} \int_{W_{0}} \sum_{n=-\infty}^{\infty} c_{n} e^{i n \mu} T^{\prime}(u, v, \mu) d \mu
$$

where

$$
T^{\prime}(u, v, \mu)=T(u, v, \mu)\left[x_{u} \cos (\mu)+y_{u} \sin (\mu)\right]
$$

$T^{\prime}$ can be expanded as a Fourier series:

$$
T^{\prime}(u, v, \mu)=\sum_{n=-\infty}^{\infty} \tau_{n}^{\prime} e^{i n \mu}
$$


Inserting Eq.(3.39) into Eq.(3.37) and integrating, yields

$$
V^{i n t}=\sum_{m=-\infty}^{\infty} c_{m} \tau_{-m}^{\prime} .
$$

A numerically efficient way to generate all of the quantities that are needed to satisfy penetrable fluid boundary conditions at the surface of the scatterer, the interior and exterior pressure fields, and the interior and exterior normal particle velocities has been developed. This representation is numerically efficient for several reasons. One is that Hankel functions no longer have to be calculated for every integration point along the boundary. A second is that by equally spacing integration points in the new angular variable $v$, points along the surface that change rapidly are sampled at a higher spatial rate. so fewer integration points are required than if points were equally spaced in cylindrical coordinates .

It has also been discussed how to choose the scattered field coefficients, and a convergence criteria for the farfield scattered pressure. 


\section{Chapter 4}

\section{Numerical Results}

This chapter will begin by comparing the numerical efficiency of the FMM with various existing methods. Numerical results generated using the FMM will then be presented for a several types of cross sections and boundary conditions and compared with exact solutions, where applicable, or approximate solutions. All results involve plots of the backscattered form function as defined in Eq. (2.29) versus a dimensionless term $k$ times a characteristic dimension of the cross section. The equations used to generate all results for the FMM are given by Eq.(3.13) for rigid boundary conditions, Eq.(3.14) for soft boundary conditions, and Eq.(3.15) for penetrable fluid boundary conditions. The modal functions for the scattered and interior fields are those given in Eqs.(3.24, 3.28) and Eqs.(3.36 and 3.40), respectively.

\subsection{Numerical Efficiency}

Two numerical methods that are used to solve the problem of scattering by a noncircular cylinder are the MMM and the T-Matrix method. One similarity between both of these approaches and the FMM is that they all require the calculation a Hankel function (for each unknown coefficient) at many points along the boundary, and then 
an integration over the boundary. Therefore, the numerical efficiency of each of these methods depends on two criteria:

1. The number of unknown coefficients, and

2. the number of integration points.

For smooth scatterers that do not deviate strongly from circular, there will not be a significant difference between the numerical efficiency of the FMM and the T-Matrix or the MMM. However, in more extreme cases, the FMM can be much more efficient than the other approaches.

For example, the FMM is numerically more efficient than the T-Matrix for elongated cross sections because fewer terms are needed for the numerical integrations. This arises due to the fact that the conformal mapping used in the FMM samples points along the scatterer surface that change rapidly at a higher spatial rate. The following table shows the number of modes and integration points required for the FMM and the TMatrix for the case of a rigid 10:1 aspect ratio elliptic cylinder for $k L=5$, where $L$ is the length of the semimajor axis. Note that in order to make the T-Matrix stable, the basis functions for the surface field were expanded in terms of a Fourier series in $v$, and the Green's function in cylindrical eigenfunctions. The MMM required more terms than the computer could handle:

\begin{tabular}{||l|l|l|l||}
\hline Method & FMM & T-Matrix & MMM \\
\hline Number of Modes & 18 & 18 & $\gg 18$ \\
\hline Number of Integration Points & 120 & 800 & 800 \\
\hline
\end{tabular}

The FMM is numerically more efficient than the MMM because far fewer unknown coefficients are required for the same degree of accuracy. The MMM requires more terms is because it strictly matches the boundary condition, whereas the FMM may only be used to calculate the far field pressure. For example, the following table shows the number 
of modes and integration points required for the FMM, the T-Matrix and the MMM for the case of a rigid 2:1 aspect ratio elliptic cylinder for $k L=3$ :

\begin{tabular}{||l|l|l|l||}
\hline Method & FMM & T-Matrix & MMM \\
\hline Number of Modes & 12 & 12 & 52 \\
\hline Number of Integration Points & 100 & 100 & 100 \\
\hline
\end{tabular}

As was discussed previously the FMM and MMM are very similar, the main difference being that the weighting functions are different. Recall the FMM uses the weighting functions $\psi_{n}=e^{i n v}$, with $v$ being the angular variable in the new coordinate system that was generated from the conformal mapping. An interesting question is whether this choice of functions is better than the set of functions $\psi_{n}=e^{i n \theta}$, where $\theta$ is the angular variable in circular coordinates. If this were true the conformal mapping would be unnecesary, since the entire formulation could be performed in circular coordinates. However, in practice the FMM requires far fewer terms than by using $\psi_{n}=e^{i n \theta}$. For example, Figure (4.1) shows the backscatter from a rigid 10:1 aspect ratio elliptic cylinder for rigid boundary conditions. The solid curve shows the result using the FMM and 16 modes, and converges to the exact solution, as will be shown in the following section. The dashed curve uses the cylindrical weighting functions for the same number of modes. The dash-dotted curve used the cylindrical weighting functions using 22 modes. As can be seen from this, the cylindrical weighting functions may be used, but will require more modes than the FMM.

\subsection{Ellipse}

The elliptic cylinder is a very important check on the FMM because the exact solution is know in terms of Mathieu functions (Appendix B). For this geometry, the results from the FMM may be compared with the exact solution for both impenetrable and penetrable fluid cylinders for a wide range of aspect ratios. Results will be presented 


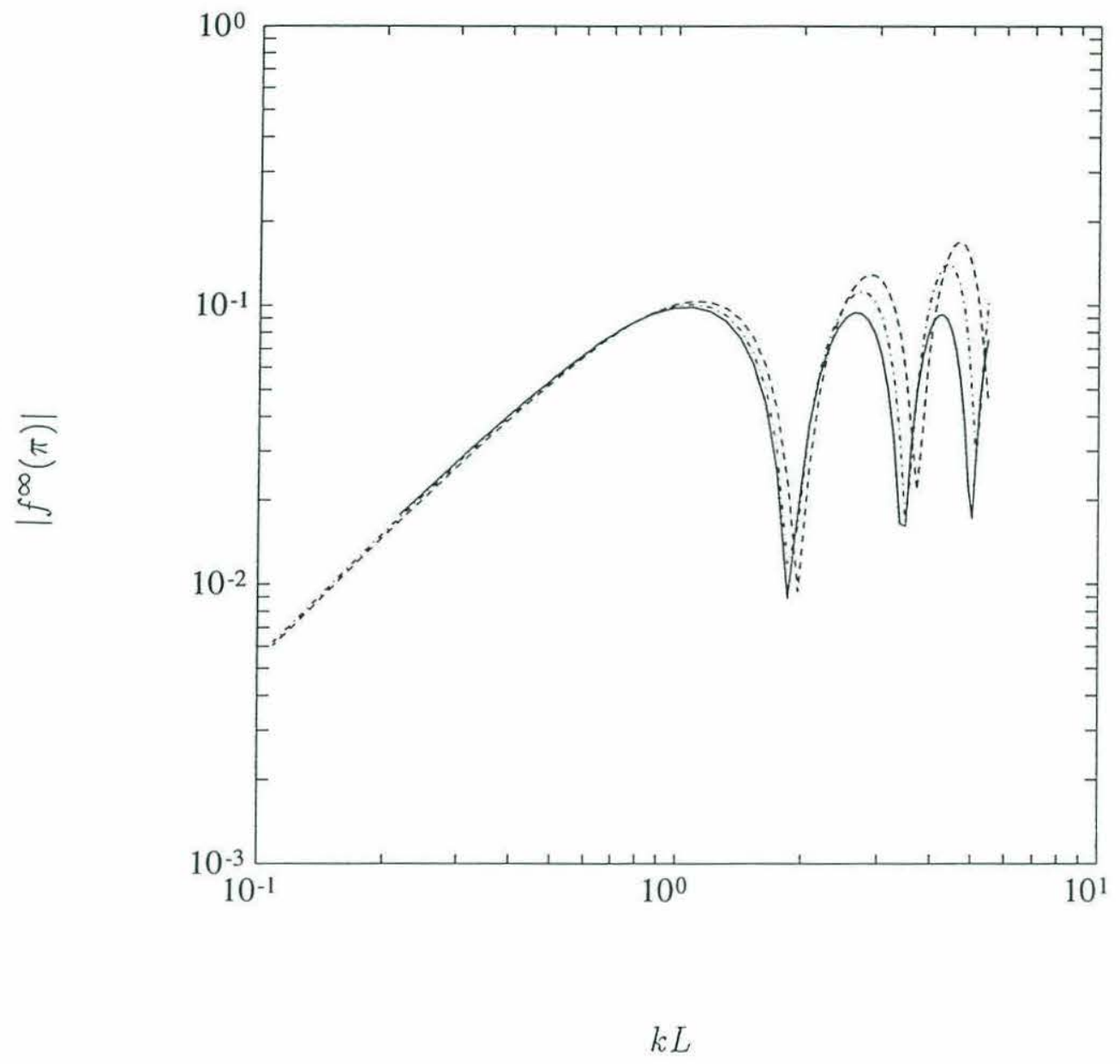

Figure 4.1: Backscattered form function of a Rigid Ten to One Ellipse: $\theta_{0}=0$. The solid curve is from the FMM using 16 modes, the dashed curve uses cylindrical weighting functions using 16 modes, and the dash-dotted curve uses cylindrical weighting functions using 22 modes. 
for a rigid 10:1 aspect ratio ellipse, and for a 2:1 aspect ratio cylinder for both rigid and penetrable boundary conditions. For all of these cases, the exact solution, and the solution from the FMM are essentially indistinguishable. Figure (4.2) shows the geometry of the elliptic cylinder for a $2: 1$ aspect ratio.

Figure (4.3) illustrates the backscatter from an approximately 10:1 aspect ratio rigid elliptic cylinder vs. $k L$ for a wave incident along the major axis. The parameters used are shown in the following table:

\begin{tabular}{||l|l||}
\hline Mapping Coefficients & $g_{-1}=1 \quad g_{1}=e^{-.2}$ \\
\hline Aspect Ratio & $10.033: 1$ \\
\hline Number of Modes & $\mathrm{M}=18$ \\
\hline Number of Integration Points & 120 \\
\hline Error & $\epsilon_{M} \leq .0001$ \\
\hline
\end{tabular}

Here both the results from the FMM and the exact result from separation of variables are plotted versus $k L$, As can be seen, the results are indistinguishable. Figure (4.4) illustrates both the exact result and the results from the FMM for a 10:1 aspect ratio ellipse under rigid boundary conditions for a plane wave incident along the minor axis. Again, the results are indistinguishable.

Figure(4.5) illustrates the backscatter from an approximately $2: 1$ aspect ratio rigid elliptic cylinder vs. wavenumber. The parameters used were:

\begin{tabular}{||l|l||}
\hline Mapping Coefficients & $g_{-1}=1 \quad g_{1}=e^{-1}$ \\
\hline Aspect Ratio & $2.164: 1$ \\
\hline Number of Modes & $\mathrm{M}=12$ \\
\hline Number of Integration Points & 100 \\
\hline Error & $\epsilon_{M} \leq .0001$ \\
\hline
\end{tabular}

Both the results from the FMM and the exact result from separation of variables are shown. Again, the results are indistinguishable. 


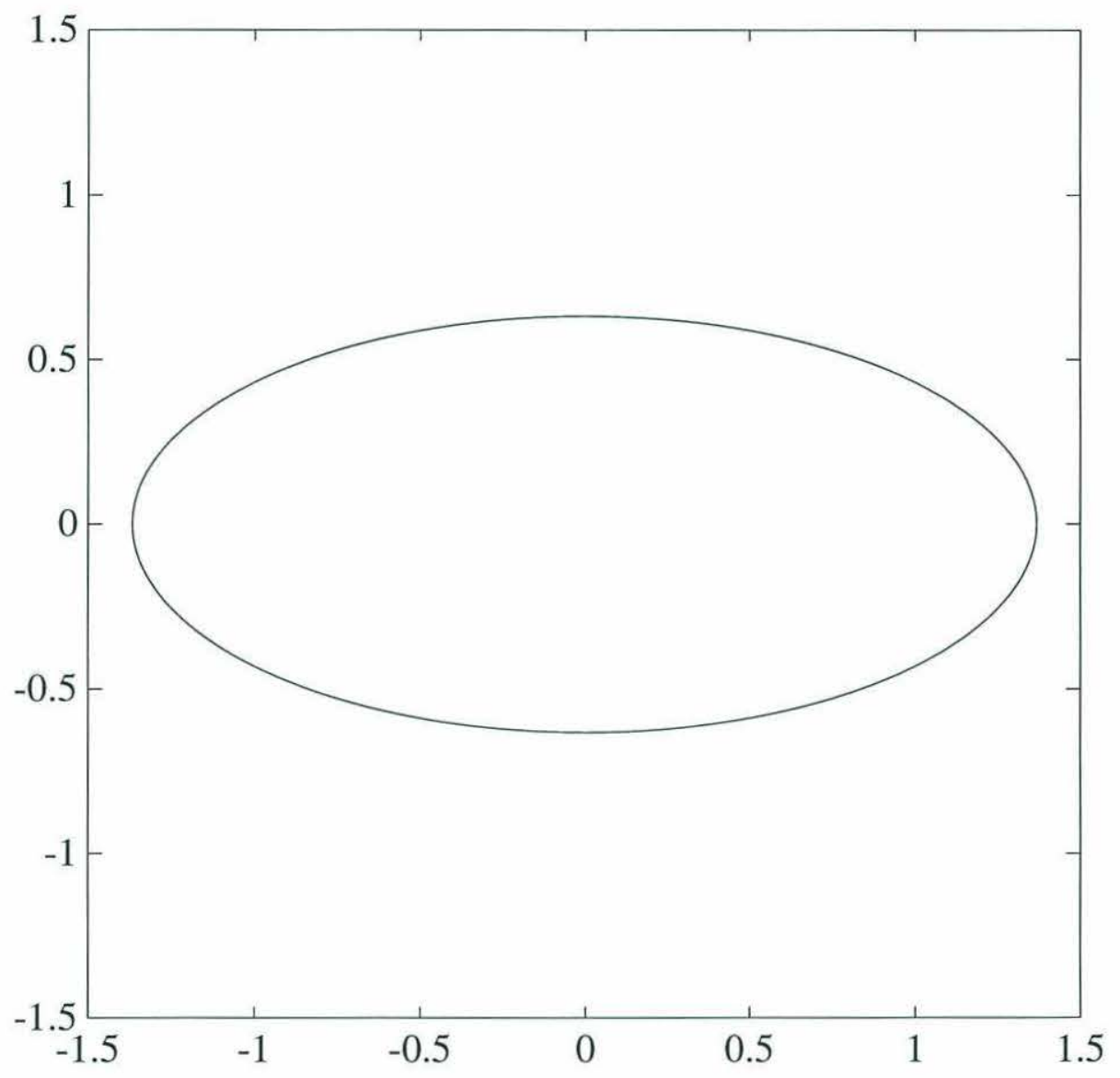

Figure 4.2: Geometry of Cross section of Elliptic Cylinder 


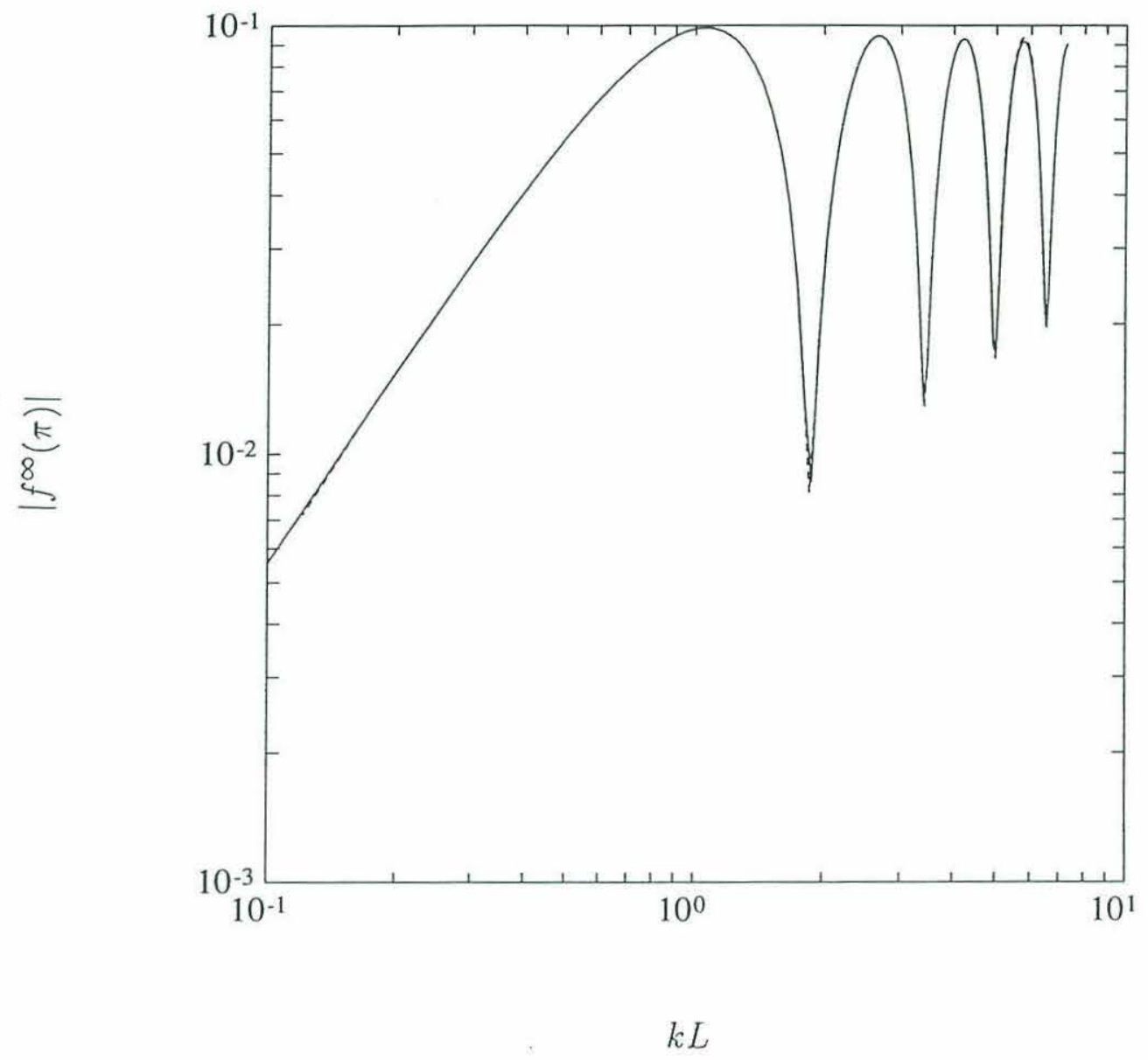

Figure 4.3: Backscattered form function of a Rigid Ten to One Ellipse: $\theta_{0}=0$ (direction of incidence along the major axis). Both exact solution and approximate solution from the FMM are plotted. 


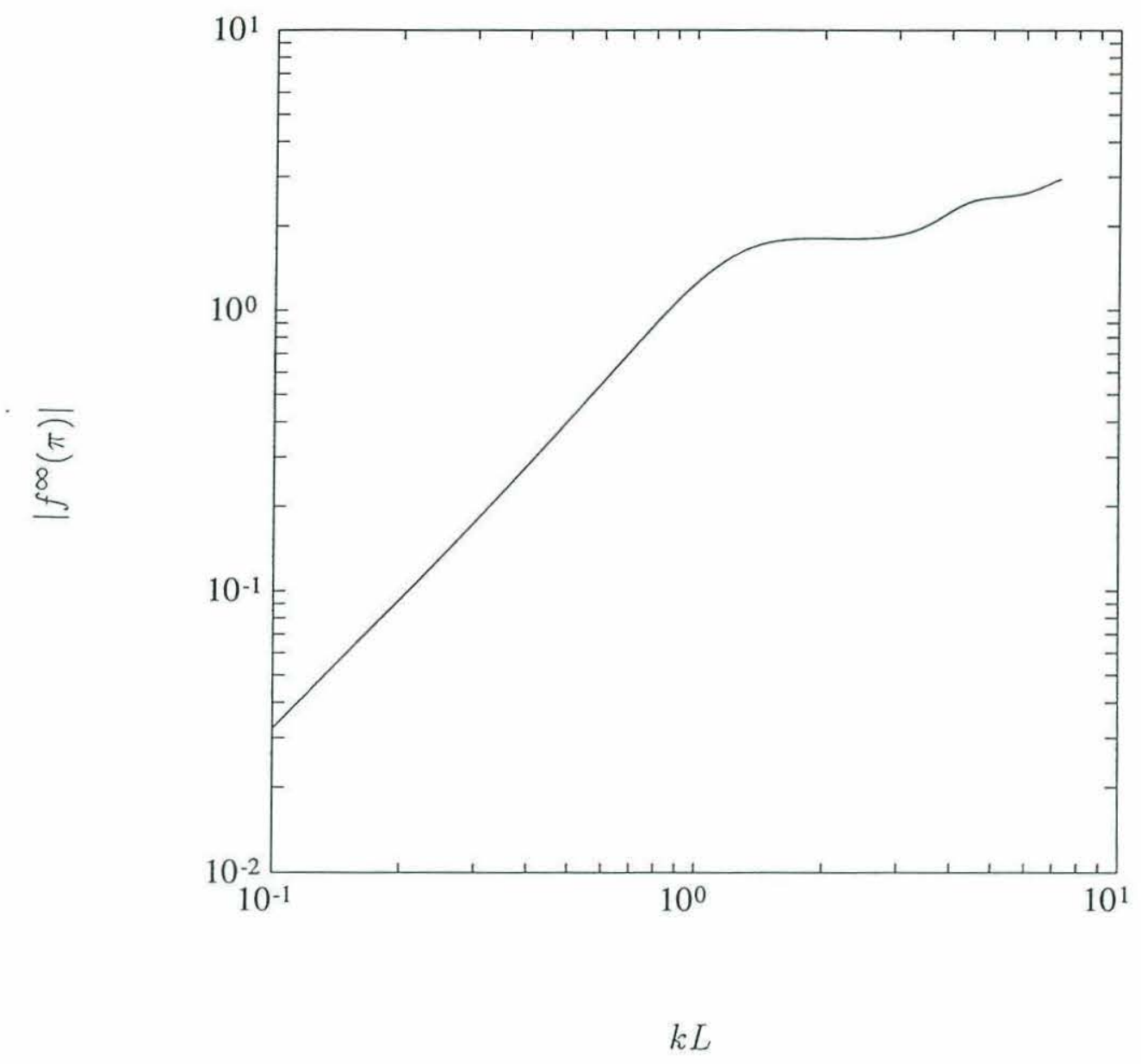

Figure 4.4: Backscattered form function of a Rigid Ten to One Ellipse: $\theta_{0}=\frac{\pi}{2}$ (direction of incidence along the minor axis). Both exact solution and approximate solution from the FMM are plotted. 


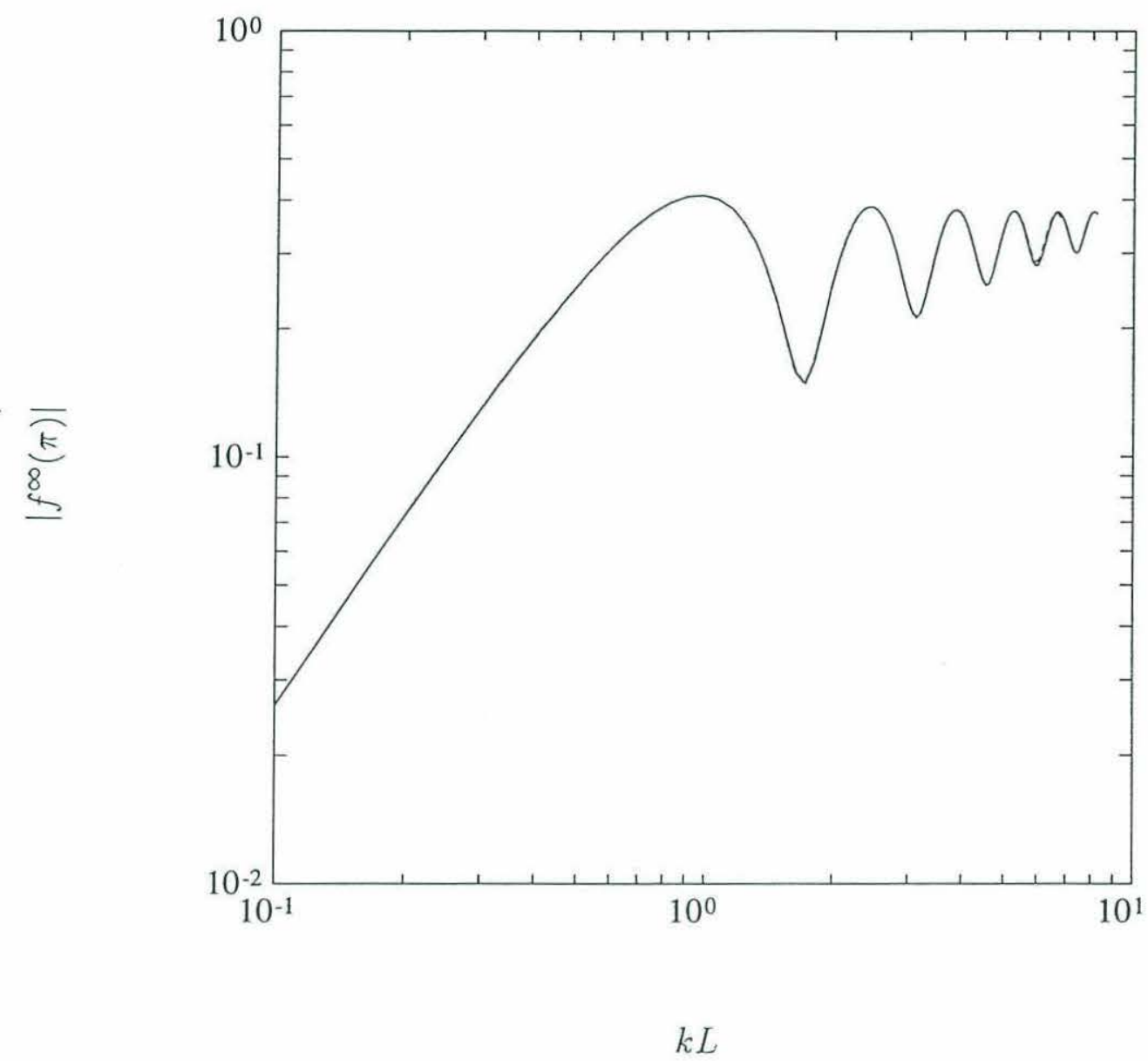

Figure 4.5: Backscattered form function of a Rigid Two to One Ellipse: $\theta_{0}=0$ (direction of incidence along the major axis). Both exact solution and approximate solution from the FMM are plotted. 
Figure (4.6) illustrates the backscatter from an approximately 2:1 aspect ratio penetrable fluid elliptic cylinder vs. wavenumber. The material parameters used were $\rho / \rho_{1}=2$, and $c / c_{1}=1.05$. Here both the results from the FMM and the exact result from separation of variables are plotted. Again, the results are indistinguishable, hence illustrating the success of the method.

\subsection{Square}

For the square cylinder, a conformal mapping will not be valid at the sharp corners. However, after truncating the mapping the corners become slightly rounded, and the mapping will be valid. Therefore, only the first few terms in the mapping will be used. The parameters used for the square cylinder are:

\begin{tabular}{||l|ll||}
\hline Mapping Coefficients & $g_{-1}=1 \quad g_{3}=-1 / 6 \quad g_{7}=1 / 56$ \\
\hline Number of Modes & $\mathrm{M}=30$ \\
\hline Number of Integration Points & 120 \\
\hline Error & $\epsilon_{M} \leq .0001$ \\
\hline
\end{tabular}

The cross section of the now rounded square cylinder appears as in Fig. (4.7). Results for the physical optics approximation as well as the FMM will be generated for the rounded square.

Figure (4.8) illustrates the backscattered form function for a plane wave incident at 45 degrees from a rigid square cylinder. The backscattered form function is plotted versus $k L$, where $\mathrm{L}$ is the length of a side of the square. The solid curve is from the FMM, and the dashed curve is from the physical optics method. Note that at low frequency the two curves do not coincide. This discrepancy is not alarming because physical optics is valid only at high frequency. As the frequency increases, the agreement between the FMM and physical optics improves. 


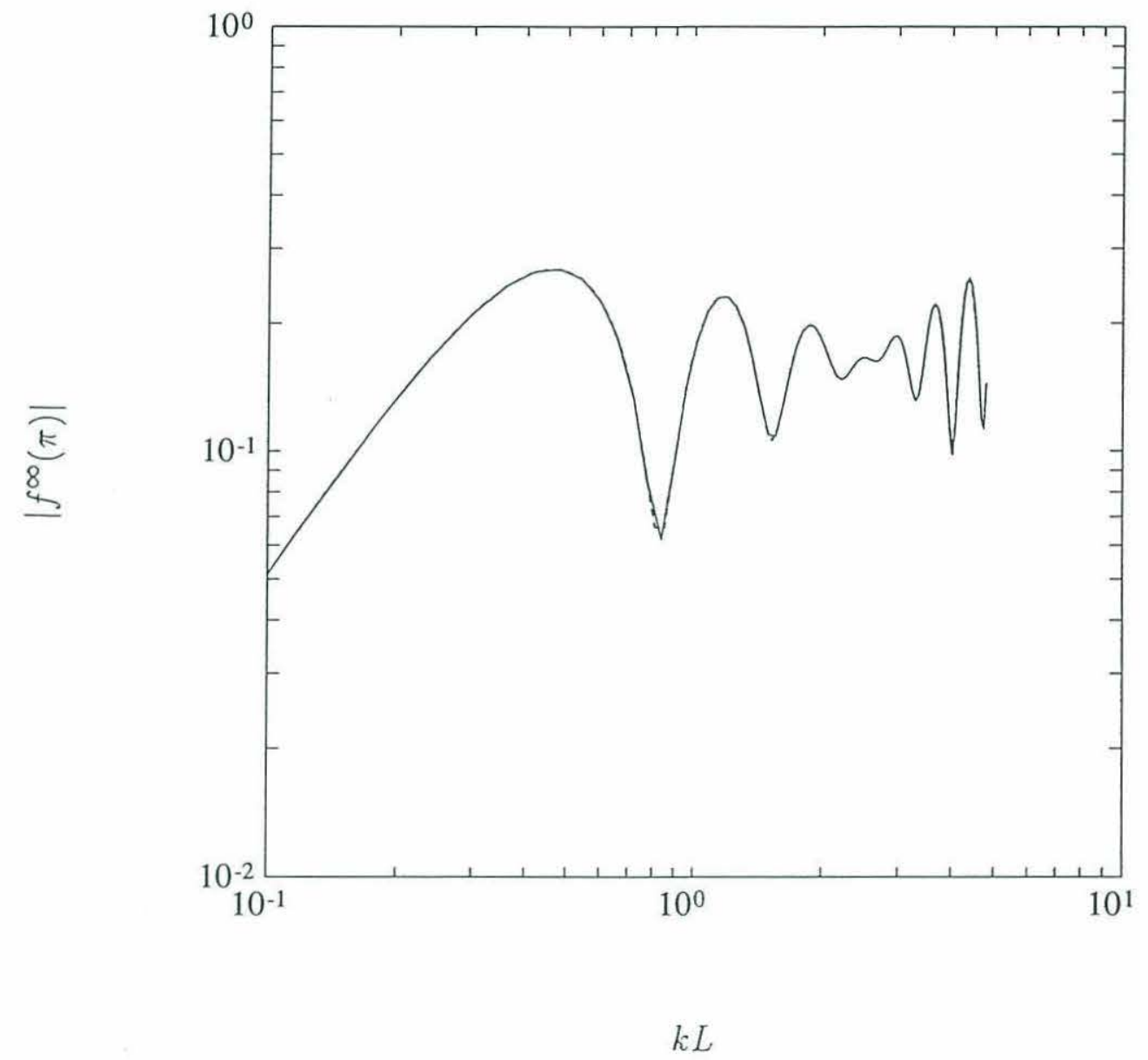

Figure 4.6: Backscattered form function of a Penetrable Two to One Ellipse: $\theta_{0}=0$ (direction of incidence along the major axis). Both exact solution and approximate solution from the FMM are plotted. The material parameters are $\frac{\rho_{1}}{\rho}=2$ and $\frac{c_{1}}{c}=1.05$ 


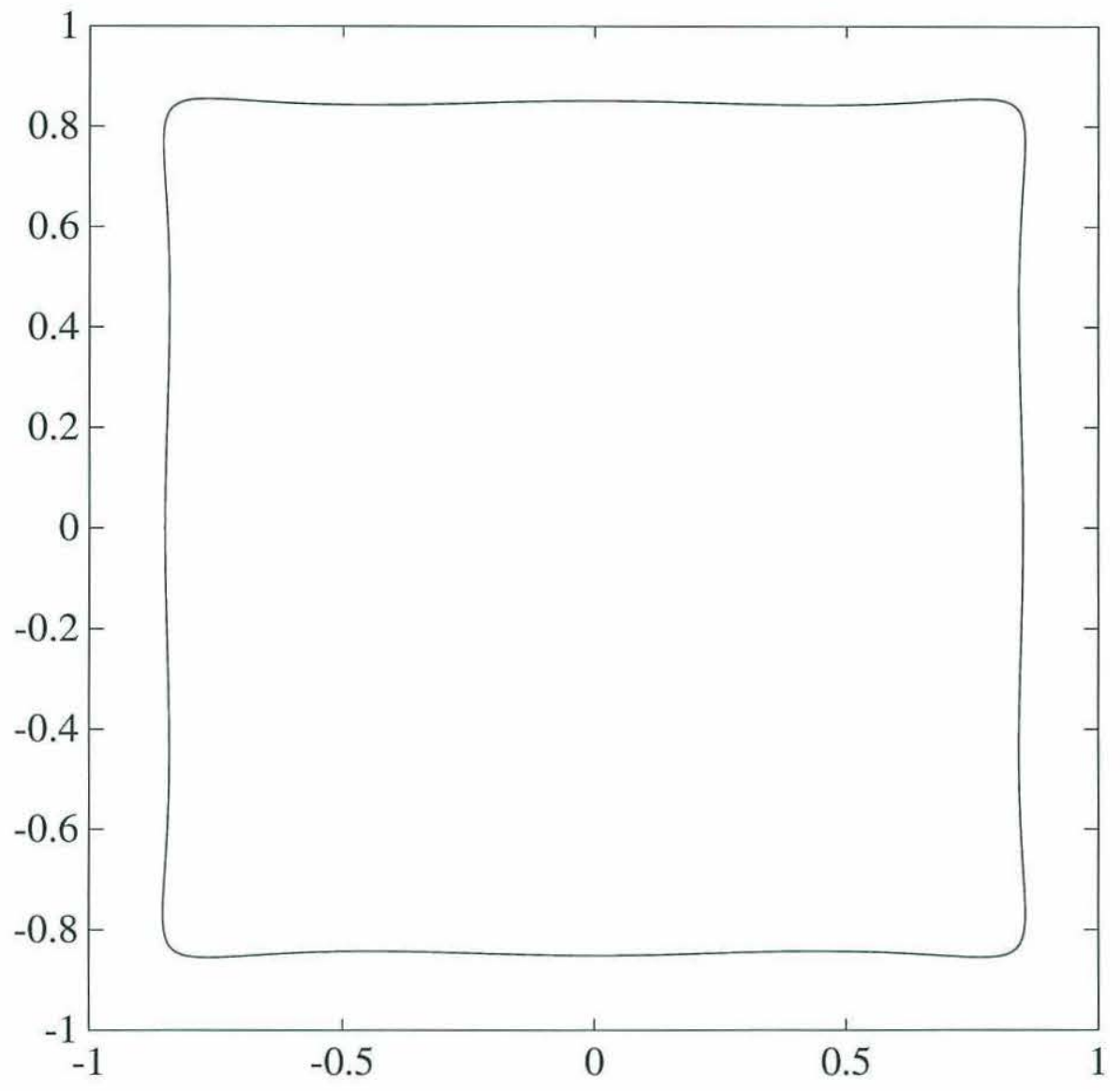

Figure 4.7: Geometry of Cross Section of a Square Cylinder 


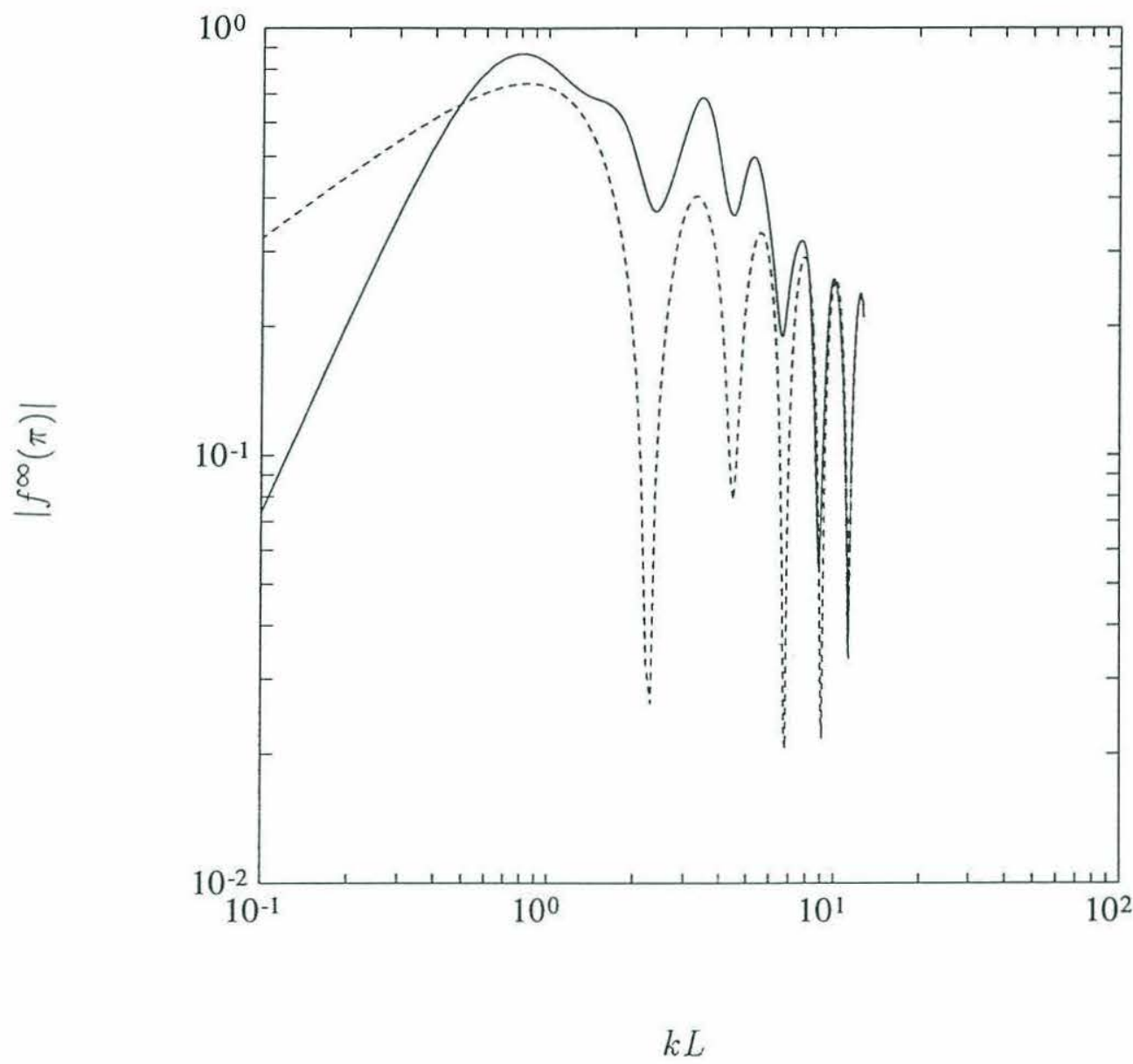

Figure 4.8: Backscattered form function of a Rigid Square : $\theta_{0}=\frac{\pi}{4}$ (direction of incidence from the corner of the square). The dashed curve is from the physical optics approximation, and the solid curve is from the FMM under rigid boundary conditions. 
Similar results are obtained for a plane wave incident at 45 degrees from a soft square cylinder, as shown in Fig. (4.9).

Figure (4.10) illustrates the backscatter of a plane wave incident at 0 degrees from a rigid square cylinder. Again, the two curves show good agreement except at low frequency, where physical optics is not valid.

For the penetrable square cylinder, there is no exact solution. However, if the scatterer is weak, that is the material properties of the scatterer are a perturbation of the surrounding fluid, the the Distorted Wave Born Approximation (DWBA) may be used. For a square cylinder with $\frac{\rho_{1}}{\rho}=1.05, \frac{c_{1}}{c}=1.05$ and a plane wave incident at 0 degrees, the scattering integral reduces to a form that may be interpreted as the interference between a reflection off the front surface of the square and a wave that is transmitted through the front face of the cylinder, reflects off the back face, and then is transmitted through the front face. By summing the contributions from these two waves, the DWBA is calculated and plotted along with results from the FMM in Fig. (4.11). The solid curve is the FMM and the dashed curve is the DWBA. Both show the dramatic oscillatory effect due to the interference between the two waves. The agreement is again excellent.

\subsection{Three Leaf Clover}

This section shows a more complex shape than considered previously. This work was conducted because it is fundamentally different than the ellipse and square in that there are concavities in the surface. The geometry is shown in Fig. (4.12). The parameters used for this shape are: 


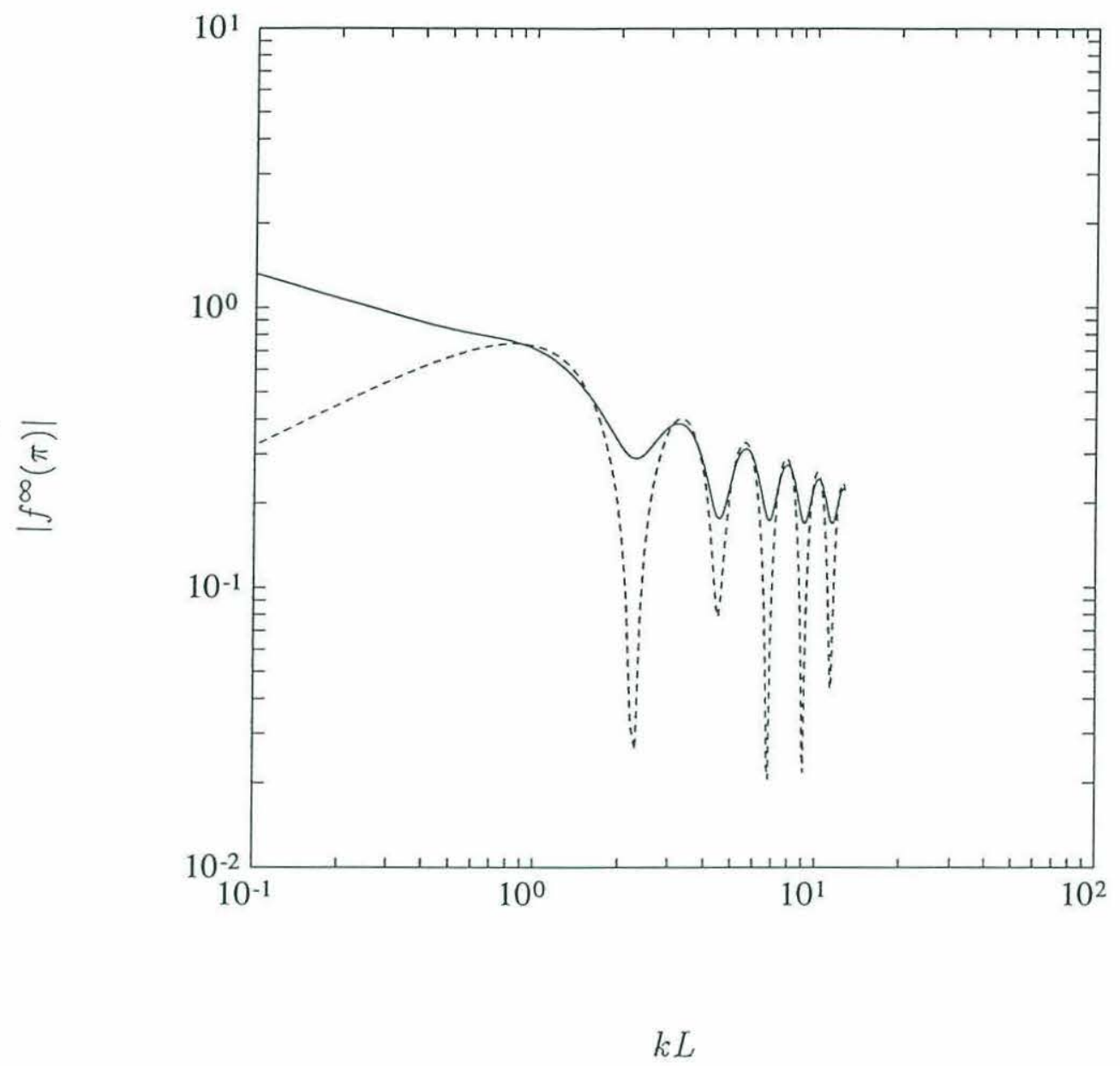

Figure 4.9: Backscattered form function of a Soft Square : $\theta_{0}=\frac{\pi}{4}$ (direction of incidence from the corner of the square). The dashed curve is is from the physical optics approximation, and the solid curve is from the FMM under soft boundary conditions 


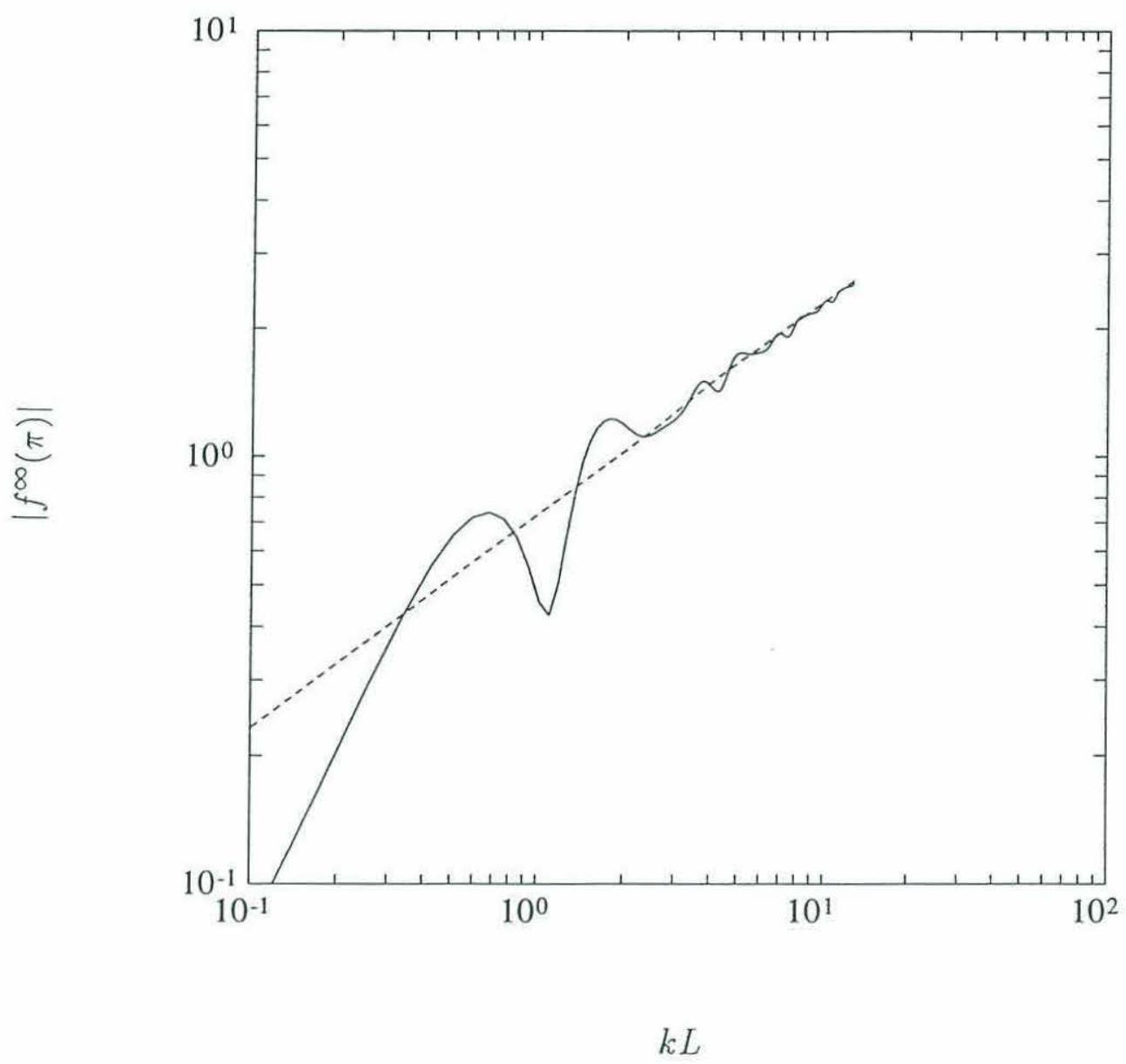

Figure 4.10: Backscattered form function of a Rigid Square : $\theta_{0}=0$ (direction of incidence from the side of the square). The dashed curve is is from the physical optics approximation, and the solid curve is from the FMM under soft boundary conditions. 


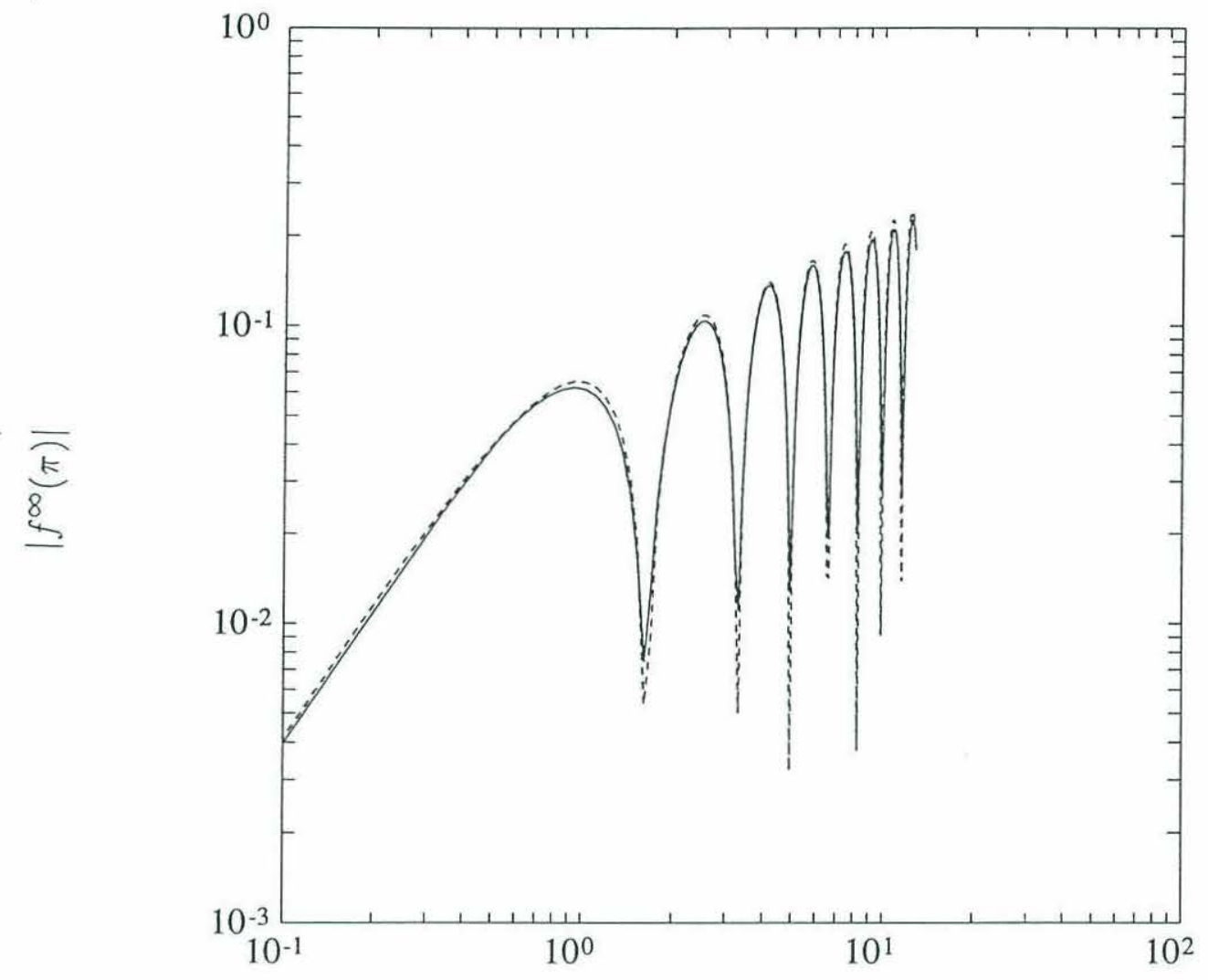

$k L$

Figure 4.11: Backscattered form function of a Weakly Scattering Square : $\theta_{0}=0$ (direction of incidence from the side of the square). The dashed curve is from the DWBA, and the solid is from the FMM. The material parameters are $\frac{\rho_{1}}{\rho}=1.05$ and $\frac{c_{1}}{c}=1.05$. 


\begin{tabular}{||l|l||}
\hline Mapping Coefficients & $g_{-1}=1 \quad g_{2}=-.2 \quad g_{5}=-.05$ \\
\hline Number of Modes & $\mathrm{M}=30$ \\
\hline Number of Integration Points & 120 \\
\hline Error & $\epsilon_{M} \leq .0001$ \\
\hline
\end{tabular}

Figure (4.13) illustrates the backscatter from a rigid clover for a plane wave incident at $\theta_{0}=\pi / 6$ compared with physical optics. The backscattered form function is plotted versus $k L$, where $L$ is the arerage radius of the surface. The dashed curve is physical optics, and the solid curve is from the FMM under rigid boundary conditions. Again, physical optics is not valid at low frequencies, so the comparison there is not expected to be close, but at high frequencies the comparison improves. For both this case, and that of the square cylinder, even at high frequencies the agreement is close but not perfect. This is to be expected because physical optics assumes that each point scatters as though it were part of an infinite plane, which is not true. It also ignores any contribution from circumferential waves, which tend to alter the structure of the scatter pattern . Figure (4.14) shows physical optics (solid curve) compared with the exact solution for a 2:1 aspect ratio elliptic cylinder. The dashed curve is for soft boundary condtions, and the dotted curve is for rigid boundary conditions. As can be seen, the physical optics method will not necesarily accurately predict the level of the nulls. 


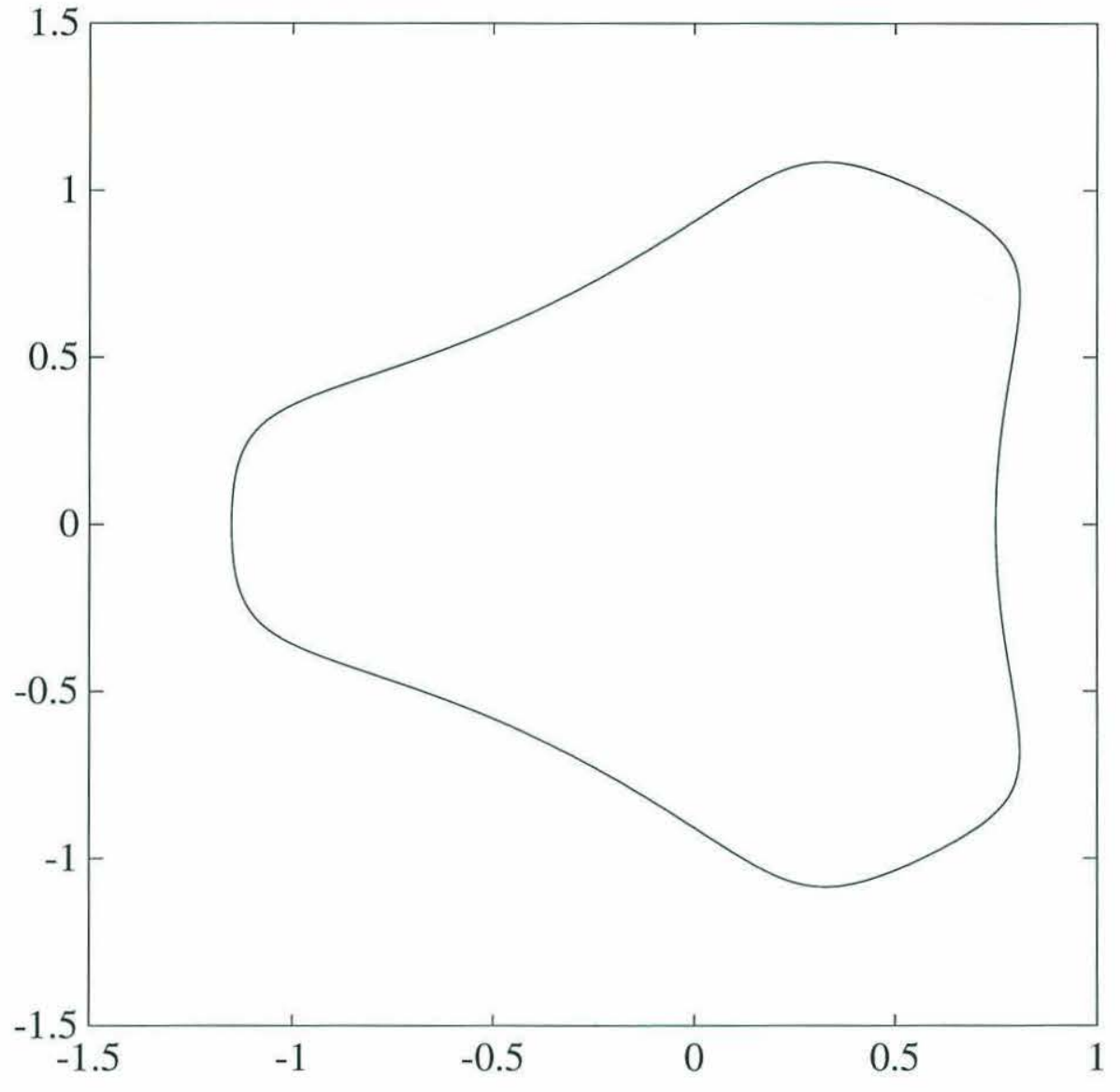

Figure 4.12: Geometry of Cross Section of Three Leaf Clover 


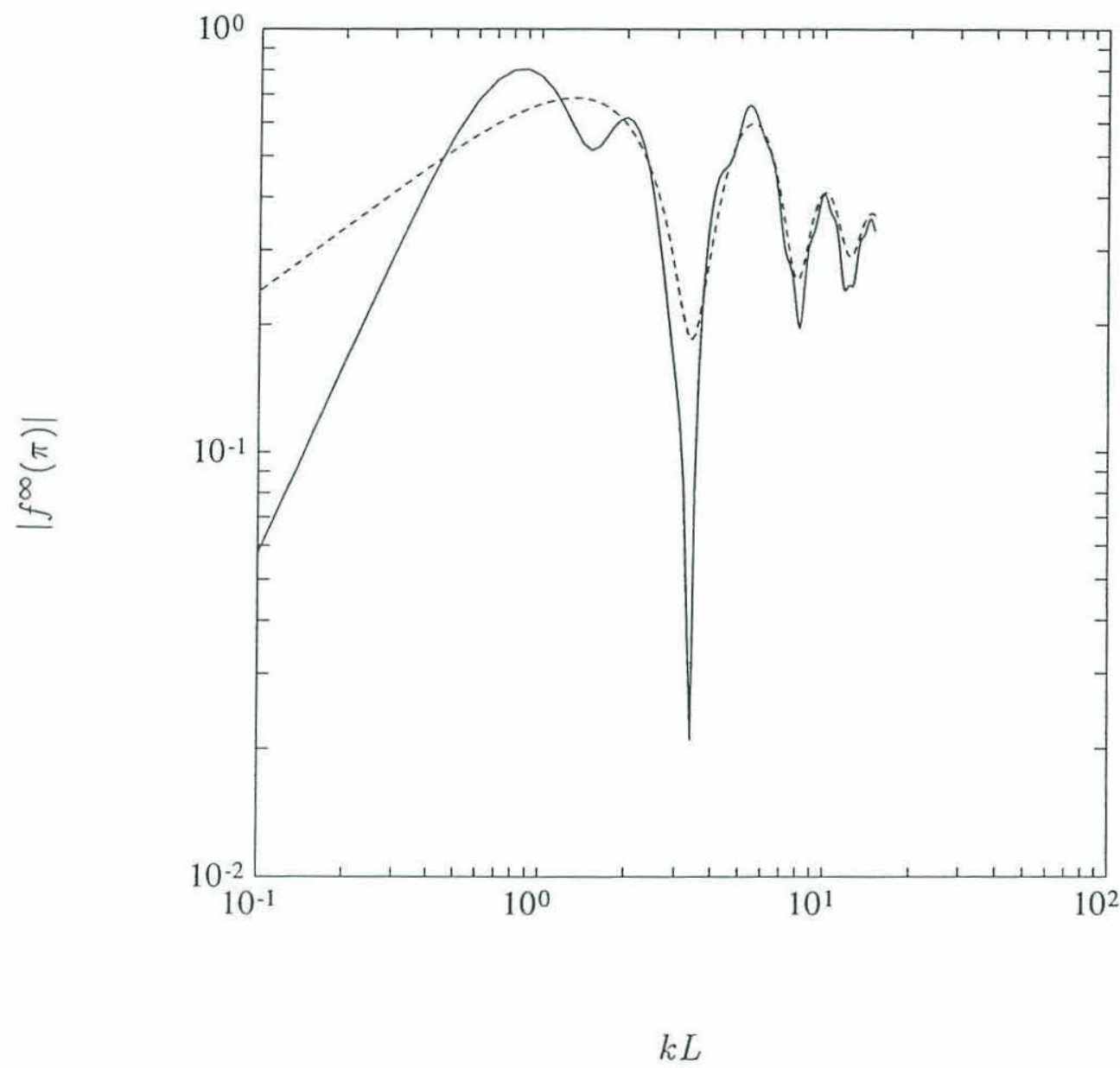

Figure 4.13: Backscattered form function of a Rigid Clover : $\theta_{0}=\pi / 6$. The dashed curve is from the physical optics approximation, and the solid curve is from the FMM under soft boundary conditions. 


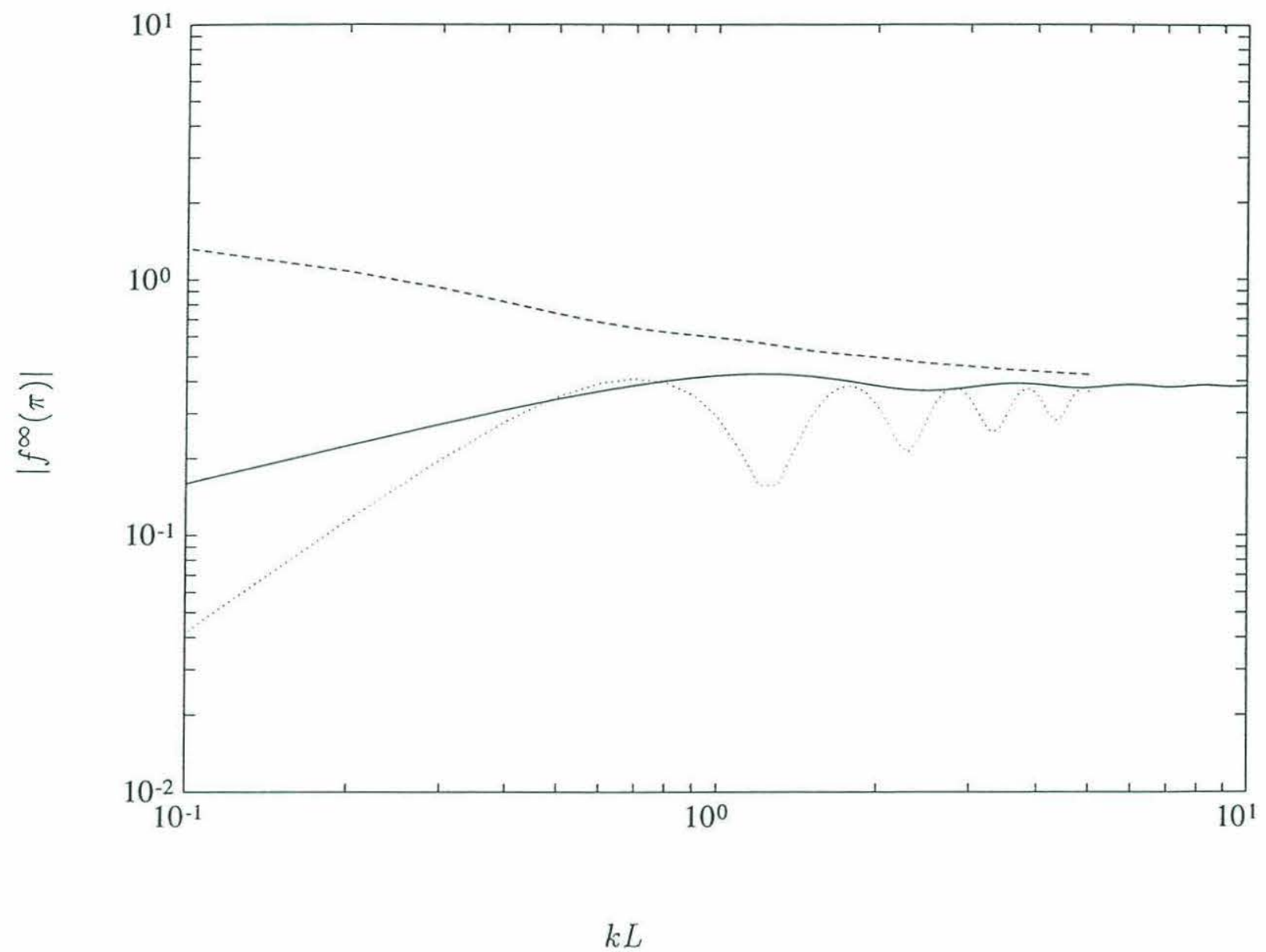

Figure 4.14: Backscattered form function from a 2:1 Ellipse. $\theta_{0}=0$. The solid curve is is from the physical optics approximation, the dashed curve is from the exact solution under soft boundary conditions, and the dotted curve is from the exact solution under rigid boundary conditions. This illustrates the fact that the physical optics solution will not necesarily accurately predict the level of the nulls. 


\section{Chapter 5}

\section{Discussion and Conclusions}

\subsection{Conclusions}

The development of a new method for calculating the farfield scattered pressure of a plane wave due to a cylinder of noncircular cross section has been presented in this thesis. The new method has been shown to be very accurate and numerically efficient for a wide range of cross sections and boundary conditions. The formulation is simple in that it does not need to be tailored to the shape of the scatterer, as does the T-Matrix. Numerical results presented for elongated cross sections, as well as cross sections with sharp edges and concavities, have shown excellent agreement with exact and approximate solutions. It has also been shown to be numerically more efficient than competing approaches when the scatterer is not close to circular.

This method most nearly resembles the MMM, in that the scattered field is described as a finite summation of nonorthogonal modal functions. The difference is in how the scattered field coefficients are chosen. In the MMM, the mean squared error on the boundary is minimized. The drawback of this approach is that in order to satisfy the boundary conditions, far more coefficients are required than are necesary to describe the farfield scattered pressure. 
It is here that the method developed in this thesis, the FMM, becomes useful. Instead of minimizing the mean squared error, the scattered field coefficients are chosen so that there is no error in the Fourier components $e^{i n v}$ for $|n| \leq N$. In practice fewer terms are required to have a convergent farfield scattered pressure than to satisfy the boundary conditions rigorously. Therefore, if nearfield quantities are not required, the FMM will in general be more efficient than the MMM.

Another benefit of this research is the fact that the conformal mapping portion may be used in conjunction with the T-Matrix to improve the range of surfaces the method can handle. For example, by expressing the surface fields in terms of the new angular variable $v$, accurate results were achieved for an elongated (elliptical) cross section using the TMatrix, where the Green's function was expressed in terms of cylindrical eigenfunctions. This improvement was due to the fact that sampling points for the numerical integrations were more rapidly sampled at the ends of the ellipse, therefore eliminating the error that causes the method to become unstable.

Although the FMM may be more efficient and general than both the MMM and T-Matrix, the major disadvantage of the FMM is that it has not been rigorously shown to converge to the true solution. Results have been presented that show excellent agreement with reference solutions. By proof of example, The author is confident that the solution is, in fact, correct. In order to use the FMM, it is necesary to change variables to a new coordinate system in which the radial coordinate $u=0$ is the scatterer surface. This is achieved through a conformal mapping, namely conformally mapping the exterior of a closed surface to a semi-infinite strip, and the interior of a closed surface to a semi-infinite strip. To the knowledge of the author, this is the first time that an efficient algorithm has been presented for doing this.

In conclusion, a new technique for solving the problem of scattering from a cylinder of noncircular cross section has been presented. This technique has proven to be accurate for a wide variety of cross sections and boundary conditions, as well as numerically 
efficient. Several examples of backscatter calculations were presented and compared to either exact or approximate solutions. As discussed in the following section, however, some interesting research remains to be done.

\subsection{Recommendations for Future Research}

There are several types of research that could follow directly from the work presented in this thesis. Since an efficient algorithm has been developed to describe the scattering from a noncircular cylinder, it would be straightforward to generate timedomain scattering data from which the physics underlying the scattering process could be studied.

Although the focus of this research has been the scattering of sound by a (penetrable) fluid cylinder, the extension to an elastic cylinder would be straightforward. For example, the shear wave that may be supported in an elastic scatterer may be accounted for by introducing another set of unknown coefficients for that new field, and then that set of coefficients would be eliminated by introducing the extra boundary condition that arises for an elastic boundary.

A third area that would be interesting to pursue would be that of a three-dimensional scatterer. This would be more difficult because conformal mapping involves only two dimensions and might not be extendable to three dimensions.

A fourth area, alluded to earlier in the thesis, involves using the fact that the Helmholtz equation in the new coordinates is the same as the Helmholtz equation in Cartesian coordinates with a spatially dependent wavenumber. Propagation of an acoustic wave in a range and depth dependent environment is an important and difficult problem, and the methods developed in this thesis may give insight into a new technique for solving this problem. 


\section{Chapter 6}

\section{Appendices}

\subsection{Appendix A: Numerical Solution of Nonlinear System of Equations}

This appendix describes how to numerically solve the set of nonlinear equations that arise from the conformal mapping procedure. The procedure is a simple extension of the Newton-Raphson procedure [35]. The Newton-Raphson method is an iterative algorithm that linearizes the nonlinear system of equations. For example, consider the one-dimensional case

$$
d(\delta)=0
$$

where $d(\delta)$ is a nonlinear function of $\delta$, and $\delta$ is the unknown. Assume an estimate of the value of the root of the equation is $\delta^{0}$, such that $d\left(\delta^{0}+h^{0}\right)=0$, where $h^{0}$ is small. This may be written as a Taylor series

$$
d\left(\delta^{0}+h^{0}\right)=d\left(\delta^{0}\right)+h^{0} d^{\prime}\left(\delta^{0}\right)+\cdots
$$

Retaining only the first term, and setting $d\left(\delta^{0}+h^{0}\right)=0$, the correction term may be solved for: $h^{0}=d\left(\delta^{0}\right) / d^{\prime}\left(\delta^{0}\right)$. The new estimate of the root thus becomes $\delta^{1}=\delta^{0}+h^{0}$. The entire procedure is now repeated, with the Mth correction term being given by 
$h^{M-1}=d\left(\delta^{M-1}\right) / d^{\prime}\left(\delta^{M-1}\right)$. In practice $\mathrm{M}$ may be chosen such that $d\left(\delta^{M}\right)$ is less than some tolerance.

This is easily generalizable to the $\mathrm{N}$-dimensional case. The problem in N-dimensions is

$$
\begin{array}{rr}
d_{1}\left(\delta_{1}, \cdots, \delta_{n}\right)= & 0 \\
\vdots & \vdots \\
d_{n}\left(\delta_{1}, \cdots, \delta_{n}\right)= & 0
\end{array}
$$

Following the same procedure as in the one-dimensional case, the Mth correction vector is obtained from solving the following:

$$
\underline{d}\left(\underline{\delta}^{M-1}\right)+\underline{J}^{M-1} \underline{h}^{M-1}=0
$$

where $\underline{h}^{M-1}$ is the $M$ th correction vector, and $\underline{J}^{M-1}$ is the Jacobian matrix whose elements are given by

$$
J_{i, j}^{M-1}=\left.\frac{\partial d_{i}}{\partial \delta_{j}}\right|_{\delta^{M-1}}
$$

Solving for $\underline{h}^{M-1}$, a new estimate for the solution vector becomes:

$$
\underline{\delta}^{M}=\underline{\delta}^{M-1}+\underline{h}^{M-1}
$$

Again, this process is iterated until the maximum of $\underline{d}\left(\underline{\delta}^{M}\right)$ is less than some tolerance.

Note here that most of the computation time in the Newton-Raphson method is in calculating the Jacobian matrix. The modified Newton-Raphson avoids this by calculating the Jacobian only for the original estimate of the solution vector $\underline{\delta}^{0}$ and solving the following equation

$$
\underline{d}\left(\underline{\delta}^{M-1}\right)+\underline{J}^{0} \underline{h}^{M-1}=0
$$

Using this method will generally require a larger $\mathrm{M}$, but it is much faster because the Jacobian only has to be calculated once. 
The convergence in either method will be very fast provided the initial guess $\underline{\delta}^{0}$ is close to the true solution. For shapes that are nearly circular, a reasonable initial guess is that $\underline{\delta}^{0}=\underline{0}$. For shapes that differ considerably from circular, an extension of Newton-Raphson must be tried. Recall that on the surface

$$
x+\left.i y\right|_{\text {surface }}=a e^{i \phi}+\sum_{n=1}^{\infty}\left[R_{n}^{*} e^{i(1+n) \phi}+R_{n} e^{i(1-n) \phi}\right]
$$

If the $R_{n}$ are not small, the new procedure is to divide all $R_{n}$ by some suitably large integer $L$, leading to the sequence of problems

$$
x+\left.i y\right|_{\text {surface }}=a e^{i \phi}+\frac{l}{L} \sum_{n=1}^{\infty}\left[R_{n}^{*} e^{i(1+n) \phi}+R_{n} e^{i(1-n) \phi}\right]
$$

The problem for $l=1$ is solved using the intial guess of $\underline{\delta}^{0}=\underline{0}$. Once the $\underline{\delta}^{M}$ have been solved for, these values are used as the initial guess for $l=2$. The procedure is then continued until $l=L$, which is the original problem. 


\subsection{Appendix B: Equations used in Numerical comparisons}

This appendix will describe the equations used in generating the numerical results various existing solutions. The results are used in the main text for comparison with the FMM. Included are descriptions of the 1) exact solutions which involves separation of variables for the two surfaces which are applicable to this thesis, circular and elliptical, for both penetrable and impenetrable scatterers, 2) the physical optics approximation for high frequency impenetrable scatterers, and 3) the Distorted Wave Born Approximation for weakly scattering (highly penetrable) bodies for all frequencies.

\subsubsection{Exact Solutions for Separable Geometries}

As discussed earlier, the conformal mapping changes the Helmholtz equation in Cartesian coordinates to a new Helmholtz equation

$$
P_{u u}+P_{v v}+k^{2} \mathcal{F}(u, v) P=0
$$

where $\mathcal{F}(u, v)$ depends on the specific mapping. If $\mathcal{F}(u, v)$ is a sum of a function of $u$ and a function of $v$, then the equation is said to be separable. That means that it may be solved by separation of variables. The equation becomes:

$$
P_{u u}+P_{v v}+k^{2}[f(u)+g(v)] P=0
$$

By assuming that the solution is a product of a function of $u$ and a function of $v$, $P=U(u) V(v)$, and insertng this into Eq.(6.2), leaves

$$
\frac{U_{u u}}{U}+k^{2} f(u)=-\left[\frac{V_{v v}}{V}+k^{2} g(v)\right]
$$

Since the left side of this equation is a function of $u$ only, and the right side is a function of $v$ only, each side must be constant. This is known as a separation constant and is denoted by $\gamma$. The equation now becomes

$$
\frac{U_{u u}}{U}+k^{2} f(u)=-\left[\frac{V_{v v}}{V}+k^{2} g(v)\right]=\gamma
$$


This may now be rewritten as two equations

$$
\begin{aligned}
V_{v v}+k^{2} g(v) V+\gamma V & =0 \\
U_{u u}+k^{2} f(u) U-\gamma U & =0
\end{aligned}
$$

These are ordinary differential equations and thus may be solved rigorously. In general the first of these equations has a distinct solution for infinitely many values of $\gamma$. Each of these values $\gamma_{m}$ is called a characteristic value or eigenvalue, and the corresponding solution $V_{m}(v)$ is called an eigenfunction. Once the eigenvalue has been determined, the second equation may be solved to yield $U_{m}(u)$. The total solution is then a summation of each of these solutions, i.e.

$$
P=\sum_{m=-\infty}^{\infty} b_{m} V_{m}(v) U_{m}(u)
$$

\section{Circular}

The simplest separable geometry is a circular boundary. For this case, $f(u)=e^{2 u}$, and $g(v)=0$. The resulting ordinary differential equations become

$$
\begin{array}{r}
V_{v v}+\gamma V=0 \\
U_{u u}+k^{2} e^{2 u} U-\gamma U=0
\end{array}
$$

Solving these differential equations yields the following solutions

$$
P^{e x t}=\sum_{m=-\infty}^{\infty}\left[a_{m} e^{i m v} J_{m}\left(k e^{u}\right)+b_{m} e^{i m v} H_{m}^{(1)}\left(k e^{u}\right)\right]
$$

Where the $a_{m}$ are the known incident coefficients, and $b_{m}$ are the unknown scattered field coefficients. In the interior of the scatterer, the corresponding solution is

$$
P^{i n t}=\sum_{m=-\infty}^{\infty} d_{m} e^{i m v} J_{m}\left(k_{1} e^{u}\right)
$$

Where the $d_{m}$ are unkown transmitted field coefficients. The boundary conditions are

$$
\begin{array}{r}
P^{e x t}=P^{\text {int }} \\
\hat{n} \cdot \nabla P^{e x t}=\hat{n} \cdot \nabla P^{i n t}
\end{array}
$$


Inserting Eqs.(6.8 and 6.9) into Eqs.(6.10), and utilizing the orthogonality properties of the angular eigenfunctions leaves

$$
\begin{array}{r}
a_{m} J_{m}\left(k e^{u_{0}}\right)+b_{m} H_{m}^{(1)}\left(k e^{u_{0}}\right)=d_{m} J_{m}\left(k_{1} e^{u_{0}}\right) \\
a_{m} J_{m}^{\prime}\left(k e^{u_{0}}\right)+b_{m} H_{m}^{\prime(1)}\left(k e^{u_{0}}\right)=\frac{\rho c}{\rho_{1} c_{1}} d_{m} J_{m}^{\prime}\left(k_{1} e^{u_{0}}\right)
\end{array}
$$

This is precisely the solution obtained from the formulation in Chapter 2.

\section{Elliptic}

The next simplest separable geometry is the elliptic boundary. For this case, $f(u)=$ $2 \cosh (2 u)$, and $g(v)=-2 \cos (2 v)$. The resulting ordinary differential equations become

$$
\begin{array}{r}
V_{v v}-2 k^{2} \cos (2 v) V+\gamma V=0 \\
U_{u u}+2 k^{2} \cosh (2 u) U-\gamma U=0
\end{array}
$$

The first of these equations is Mathieu's equation, and the second is Mathieu's modified equation. Note that Mathieu's equation depends on wavenumber, which is fundamentally different than the corresponding circular case, where the angular equation was independent of wavenumber. This makes no difference for the impenetrable case, but for the penetrable scatterer this means that there are no orthogonality relations between the exterior and interior angular eigenfunctions.

Solving these differential equations yields the following solutions in McLachlan's notation [10]:

$$
\begin{aligned}
P= & \sum_{m=0}^{\infty}\left[a_{2 m}^{e} C e_{2 m}(k, u) c e_{2 m}(k, v)+a_{2 m+2}^{o} S e_{2 m+2}(k, u) s e_{2 m+2}(k, v)+\right. \\
& \left.a_{2 m+1}^{e} C e_{2 m+1}(k, u) c e_{2 m+1}(k, v)+a_{2 m+1}^{o} S e_{2 m+1}(k, u) s e_{2 m+1}(k, v)\right] \\
+ & \sum_{m=0}^{\infty}\left[b_{2 m}^{e} M e_{2 m}^{(1)}(k, u) c e_{2 m}(k, v)+b_{2 m+2}^{o} N e_{2 m+2}^{(1)}(k, u) s e_{2 m+2}(k, v)+\right. \\
& \left.b_{2 m+1}^{e} M e_{2 m+1}^{(1)}(k, u) c e_{2 m+1}(k, v)+b_{2 m+1}^{o} N e_{2 m+1}^{(1)}(k, u) s e_{2 m+1}(k, v)\right]
\end{aligned}
$$


Where the $a$ are the known incident coefficients, and the $b$ are the unknown scattered field coefficients. The superscripts "e" and "o" are for even or odd solutions, and the subscripts ${ }_{2 m}, 2 m+1$ tell whether the angular function is periodic in $\pi$ or in $2 \pi$. Therefore there are 4 distinct types of Mathieu functions.

1. Even in $2 \pi: c e_{2 m+1}(k, v)$

2. Odd in $2 \pi: s e_{2 m+1}(k, v)$

3. Even in $\pi: c e_{2 m}(k, v)$

4. Odd in $\pi: s e_{2 m}(k, v)$

The $c e(k, v), s e(k, v)$ are angular Mathieu functions corresponding to cosine and sine functions, respectively. The $M e^{(1)}(k, u), N e^{(1)}(k, u)$ are radial Mathieu functions corresponding to Hankel functions of the first kind, and $\mathrm{Ce}(k, u), S e(k, u)$ are radial Mathieu functions corresponding to Bessel functions. The interior solution is

$$
\begin{aligned}
P^{i n t}= & \sum_{m=0}^{\infty}\left[d_{2 m}^{e} C e_{2 m}\left(k_{1}, u\right) c e_{2 m}\left(k_{1}, v\right)+d_{2 m+2}^{o} S e_{2 m+2}\left(k_{1}, u\right) s e_{2 m+2}\left(k_{1}, v\right)+\right. \\
& \left.d_{2 m+1}^{e} C e_{2 m+1}\left(k_{1}, u\right) c e_{2 m+1}\left(k_{1}, v\right)+d_{2 m+1}^{o} S e_{2 m+1}\left(k_{1}, u\right) s e_{2 m+1}\left(k_{1}, v\right)\right]
\end{aligned}
$$

Where the $d$ are the unknown transmitted field coefficients.

Since Mathieu functions are not available in MATLAB, they had to be written. They were coded directly from McLachlan, with the radial Mathieu functions being expanded in terms of products of Bessel functions.

For the rigid cylinder, the boundary condition is

$$
\hat{n} \cdot \nabla P^{e x t}=0
$$

Using the orthogonality relations leaves

$$
b_{2 m}^{e}=\frac{C e_{2 m}^{\prime}\left(k, u_{0}\right)}{M e_{2 m}^{\prime(1)}\left(k, u_{0}\right)} a_{2 m}^{e}
$$




$$
\begin{aligned}
& b_{2 m+1}^{e}=\frac{C e_{2 m+1}^{\prime}\left(k, u_{0}\right)}{M e_{2 m+1}^{\prime(1)}\left(k, u_{0}\right)} a_{2 m+1}^{e} \\
& b_{2 m+1}^{o}=\frac{S e_{2 m+1}^{\prime}\left(k, u_{0}\right)}{N e_{2 m+1}^{\prime(1)}\left(k, u_{0}\right)} a_{2 m+1}^{o} \\
& b_{2 m+2}^{o}=\frac{S e_{2 m+2}^{\prime}\left(k, u_{0}\right)}{N e_{2 m+2}^{\prime(1)}\left(k, u_{0}\right)} a_{2 m+2}^{o}
\end{aligned}
$$

The penetrable case is significantly more difficult. Again the boundary conditions are

$$
\begin{array}{r}
P^{e x t}=P^{i n t} \\
\hat{n} \cdot \nabla P^{e x t}=\hat{n} \cdot \nabla P^{i n t}
\end{array}
$$

Since the interior wavenumber is now different than the exterior wavenumber, ther is no longer orthogonality between the interior and exterior angular wavefunctions. By multiplyng by the exterior angular eigenfunctions and integrating, the result is a summation on the right side of the equation:

$$
\begin{gathered}
b_{2 m}^{e} M e^{(1)}\left(k, u_{0}\right)+a_{2 m}^{e} C e_{2 m}\left(k, u_{0}\right)=\sum_{n=0}^{\infty} \alpha_{n, m} d_{2 m}^{e} C e_{2 m}\left(k_{1}, u_{0}\right) \\
b_{2 m}^{e} M e^{\prime(1)}\left(k, u_{0}\right)+a_{2 m}^{e} C e_{2 m}^{\prime}\left(k, u_{0}\right)=\frac{\rho c}{\rho_{1} c_{1}} \sum_{n=0}^{\infty} \alpha_{n, m} d_{2 m}^{e} C e_{2 m}^{\prime}\left(k_{1}, u_{0}\right) \\
\alpha_{n, m}=\int_{0}^{2 \pi} c e_{2 n}\left(k, u_{0}\right) c e_{2 m}\left(k_{1}, u_{0}\right)
\end{gathered}
$$

This is equivalent to expanding each $c e_{2 m}\left(k_{1}, u_{0}\right)$ in terms of $c e_{2 m}\left(k, u_{0}\right)$, which is what Yeh[11] did. This is only for even solutions periodic in $\pi$, and there are corresponding results for the other types of solutions. These equations may be solved for $b_{2 m}^{e}$, but now each $b_{2 m}^{e}$, is coupled to all of the $a_{2 m}^{e}$, i.e.

$$
b_{2 m}^{e}=\sum_{n=0}^{\infty} \beta_{n, m} a_{2 m}^{e}
$$

Similar expressions may be derived for the remaining scattered field coefficients. 


\subsubsection{High Frequency (Physical Optics) Approximation}

The backscattered form function for the physical optics approximation is given by the following integral [19]

$$
f^{\infty}(\pi)= \pm \sqrt{\frac{i k}{2 \pi}} \int_{A}(\hat{k} \cdot \hat{n}) e^{i 2(\vec{k} \cdot \vec{r})} d A
$$

where $\hat{k}$ is a unit vector in the direction of the incident wave, $\hat{n}$ is the unit normal vector, $\vec{k}$ is the incident wavevector, $\vec{r}$ is the position vector of each point on the surface, $d A$ is a differential surface element, and $A$ is the ensonified area.

Because the surface normal $\vec{n}$ is very easy to calculate in the new coordinate system, the integral will be evaluated in this coordinate system. Using the properties of the new coordinate system, the quantities in the integral become:

$$
\begin{array}{r}
\hat{k} \cdot \hat{n}=\frac{1}{h_{u}}\left[x_{u} \cos \left(\theta_{0}\right)+y_{u} \sin \left(\theta_{0}\right)\right] \\
\vec{k} \cdot \vec{r}=k\left(x \cos \left(\theta_{0}\right)+y \sin \left(\theta_{0}\right)\right. \\
d A=h_{u} d v
\end{array}
$$

Inserting these into the above integral leaves

$$
f^{\infty}(\pi)= \pm \sqrt{\frac{i k}{2 \pi}} \int_{A}\left[x_{u} \cos \left(\theta_{0}\right)+y_{u} \sin \left(\theta_{0}\right)\right] e^{i 2 k\left(x \cos \left(\theta_{0}\right)+y \sin \left(\theta_{0}\right)\right)} d v
$$

All of the quantities appearing in the above integral are easily calculated once the mapping coefficients are known. For example, $x$ and $y$ are found by taking the real and imaginary parts of the mapping function:

$$
x+i y=g_{-1} e^{i v}+\sum_{n=0}^{\infty} g_{n} e^{-i n v} .
$$


$x_{u}$ and $y_{u}$ may be found by taking the real and imaginary parts of the derivative of the mapping function with respect to $u$ :

$$
x_{u}+i y_{u}=g_{-1} e^{i v}-\sum_{n=0}^{\infty} n g_{n} e^{-i n v} .
$$

The mapping coefficients used for the shapes in this thesis have already been determined elsewhere in this thesis, and are summarized as follows:

- 10:1 ellipse : $g_{-1}=1, g_{1}=e^{-.2}$

- $2: 1$ ellipse : $g_{-1}=1, g_{1}=e^{-1}$

- square : $g_{-1}=1, g_{3}=-\frac{1}{6}, g_{7}=\frac{1}{56}$

- clover : $g_{-1}=1, g_{2}=-.2, g_{5}=-.05$

\subsubsection{Distorted Wave Born Approximation}

For weakly scattering objects, that is scatterers whose material properties are a perturbation of the surrounding fluid, the Born approximation assumes the total field on the scatterer surface to be the incident field, and then uses Green's theorem to generate the scattered pressure by integrating over the surface. An extension to the Born approximation found using the T-Matrix is given by Waterman [27]. His result is for three-dimensional scatterers, but for two dimensions it is given by:

$$
P^{s c a t}(\pi) \rightarrow \frac{e^{i k r}}{\sqrt{r}} \frac{\sqrt{k}}{4}\left[\left(\rho_{1}-\rho\right)+\left(c_{1}-c\right)\right] \int_{S} e^{i 2 \overrightarrow{k_{i}} \cdot \vec{r}} d S
$$

where $d S$ is differential surface element. For the square cylinder and plane wave incident along the side of the square, performing this integration yields

$$
\int_{S} e^{i 2 \vec{k}_{i} \cdot \vec{r}} d S=L e^{i k L}\left[1-e^{-i 2 k L}\right]
$$


The physical interpretation of this result is as follows: the first term in the brackets represents the reflection of the incident wave off the front face. The second term represents a wave that is transmitted through the front face of the scatterer, is reflected off the back face, and then is transmitted back through the front face. This can be seen from the fact that the phase of the transmitted wave is $k 2 L$, and the length of the path described above is $2 L$. However, the wavenumber in the scatterer is not $k$, but $k_{1}$. The Distorted Wave Born Approximation is found by accounting for this fact. That is, the wave that is transmitted through the cylinder will have a wavenumber $k_{1}$, where $k_{1}=k \frac{c}{c_{1}}$. Replacing $k$ by $k_{1}$ in the transmitted term yields for the farfield pressure:

$$
P^{s c a t}(\pi) \rightarrow \frac{e^{i k r}}{\sqrt{r}} \frac{\sqrt{k}}{4}\left[\left(\rho_{1}-\rho\right)+\left(c_{1}-c\right)\right] L e^{i k L}\left[1-e^{-i 2 k_{1} L}\right]
$$

In order to apply this method to a general scatterer, it is necesary to be able to evaluate the original integral in closed form, and then to separate out the contributions of the waves that are propagating on the interior of the scatterer. In general this is not possible, so this method can only be applied in certain simple cases. 


\section{Bibliography}

[1] T.B.A. Senior J.J. Bowman and P.L.E. Uslenghi. Electromagnetic and acoustic scattering by simple shapes. Hemispher, New York, 1987.

[2] V.K. Varadan V.V. Varadan, A. Lakhtakia. Comments on recent criticism of the t-matrix method. J. Acoust. Soc. Am., 84:2280-2283, 1988.

[3] R.H. Hackman and D.G. Todoroff. An application of the spheroidal-coordinatebased transition matrix: The acoustic scattering from high aspect ratio solids. J. Acoust. Soc. Am., 78:1058-1071, 1985.

[4] P.M. Morse and H. Feshbach. Methods of Theoretical Physics, volume 1, chapter 5. McGraw-Hill, New York, 1953.

[5] J.J. Faran Jr. Sound scattering by solid cylinders and spheres. J. Acoust. Soc. Am., 23:405-418, 1951.

[6] H. Uberall. Physical Acoustics, volume 10, pages 1-60. Academic, New York., 1973.

[7] L.M. Lyamshev. The scattering of sound by elastic cylinders. Sov. Phys. Acoust., 5:56-61, 1959.

[8] Junger. Sound scattering by thin elastic shells. J. Acoust. Soc. Am., 24:366-373, 1952.

[9] Philip M. Morse and Pearl J. Rubenstein. The diffraction of waves by ribbons and by slits. Physical Review, 54:895-898, 1938. 
[10] N.W McLachlan. Theory and applications of Mathieu functions. Dover, New York, 1964.

[11] C. Yeh. The diffraction of waves by a penetrable ribbon. J. Math. Phys., 4:65-71, 1963.

[12] Porter. Electromagnetic Wave Theory, pages 919-929. Pergamon, Oxford., 1965.

[13] V.K. Varadan T.A.K. Pillai, V.V. Varadan. Sound scattering by rigid and elastic elliptical cylinders in water. J. Acoust. Soc. Am., 72:1032-1037, 1982.

[14] C. Yeh. Perturbation method in the diffraction of electromagnetic waves by arbitrarily shaped penetrable obstacles. J. Math. Phys., 6:2008-2013, 1965.

[15] V.A. Erma. An exact solution for the scattering of electromagnetic waves from conductors of arbitrary shape.I.Case of cylindrical symmetry. Phys Rev., 173:1243$1257,1968$.

[16] Robert G. Kouyoumjian. Asymptotic high-frequency methods. Proceedings of the IEEE, 3:864-876, 1965.

[17] H. Ikuno and L.B. Felsen. Real and complex rays for scattering from a target with inflection points. Radio Science, 22:952-958, 1987.

[18] H. Ikuno and L.B. Felsen. Complex rays in transient scattering from a smooth targets with inflection points. IEEE Trans. Antennas Propagat., 36:1272-1279, 1988.

[19] Guillermo C. Gaunaurd. Sonar cross sections of bodies partially insonified by finite sound beams. IEEE J. Ocean. Eng, 10:213-230, 1985.

[20] J.B. Keller. Diffraction by a convex cylinder. IRE Trans. Ant. and Prop, 4:312-321, 1956.

[21] Yung Ming Chen. Diffraction by a smooth transparent object. J. Math. Phys., 5:820-832, 1964. 
[22] Robert G. Kouyoumjian. A uniform geometrical theory of diffraction for an edge in a perfectly conducting surface. Proceedings of the IEEE, 11:1448-1461, 1974.

[23] V.A. Fock. Electromagnetic diffraction and propagation problems. Pergamon, Oxford, 1965.

[24] W. Tobocman. Calculation of acoustic wave scattering by mean of the Helmholtz integral equation.i. J. Acoust. Soc. Am., 76:599-607, 1984.

[25] L. Shafai. An improved integral equation for the numerical solution of twodimensional diffraction problems. Canadian Journal of Physics, 48:954-963, 1970.

[26] Eikichi Yamashita. Analysis methods for electromagnetic wave problems, chapter 2. Artech House, Boston, 1990.

[27] P.C. Waterman. New formulation of acoustic scattering. J. Acoust. Soc. Am., 45:1417-1429, 1968.

[28] R.H.T. Bates and D.J.N. Wall. Null field approach to scalar diffraction I. General method. Phil. Trans. R. Soc. Lond. Ser.A, 287:45-78, 1977.

[29] V.K. Varadan A. Lakhtakia, V.V. Varadan. Iterative extended boundary condition method for scattering by objects of high aspect ratios. J. Acoust. Soc. Am., 76:906$912,1984$.

[30] H. Ikuno and K. Yasuura. Numerical calculation of the scattered field from a periodic deformed cylinder using the smoothing process on the mode matching method. Radio Science, 13:937-946, 1978.

[31] Eikichi Yamashita. Analysis methods for electromagnetic wave problems, chapter 4. Artech House, Boston, 1990.

[32] A. Sommerfeld. Partial Differential Equations in Physics, chapter 4. Academic Press, New York, 1949. 
[33] Werner G. Neubauer. Acoustic Reflection from Surfaces and Shapes, chapter 4. Naval Research Laboratory, Washington, D.C., 1986.

[34] Francis B. Hildebrand. Advanced Calculus for Applications, chapter 6, pages 306313. Prentice-Hall, Inc, Englewood Cliffs, New Jersey, 1976.

[35] Gilbert Strang. Introduction to Applied Mathematics, chapter 5, pages 373-380. Wellesley-Cambridge Press, Wellesley, MA, 1986. 